\title{
Microtubule buckling in an elastic matrix with quenched disorder
}

\author{
Cheng-Tai Lee and Eugene M. Terentjev ${ }^{1}$ \\ Cavendish Laboratory, University of Cambridge, J.J. Thomson Avenue, Cambridge, CB3 OHE, \\ U.K.
}

The intracellular elastic matrix has been recognized as an important factor to stabilize microtubules and increase their critical buckling force $P_{c}$ in vivo. This phenomenon was qualitatively explained by the Winkler model, which investigated buckling of a filament embedded in a homogeneous elastic medium. However, the assumption of homogeneity of the matrix in Winkler's, and other advanced models, is unrealistic inside cells, where the local environment is highly variable along the filament. Considering this to be a quenched-disorder system, we use a Poisson distribution for confinements, and apply the replica technique combined with the Gaussian variational method to address the buckling of a long filament. The results show two types of filament buckling: one corresponding to the first-order, and the other to a continuous second-order phase transition. The critical point, i.e. the switch from first- to second-order buckling transition, is induced by the increase in disorder strength. We also discover that this random disorder of the elastic environment destabilizes the filament by decreasing $P_{c}$ from the Winkler result, and the matrix with stronger mean elasticity has a stronger role of disorder (inhomogeneity). For microtubules in vivo, buckling follows the discontinuous first-order transition, with $P_{c}$ reduced to the fraction between 0.9 and 0.75 of the Winkler prediction for the homogeneous elastic matrix. We also show that disorder can affect the force-displacement relationship at non-zero temperature, while at zero temperature this effect vanishes.

\section{INTRODUCTION}

Microtubules (MTs) are rigid linear protein filaments with a large persistence length, of around millimeters, while their total length can vary from nanometers to hundreds of microns. In cells, MTs, together with actin and intermediate filaments, form a cytoskeleton (CSK) network that maintains the cell shape and mechanical response. As the tensegrity model suggested, MTs act as components which resist external compression ${ }^{[12}$ in the overall CSK matrix. It is therefore essential to understand the mechanical response of MTs to the applied compressive forces. Previous experiments and simulations showed that MT buckling is an almost in-plane process 5 . The basic mechanical response of an initially straight filament under a compression force $P$ (see Fig. 1) is described by the energy functiona $\mathrm{I}^{\sqrt{5}}$ :

$$
H_{E}=\int_{0}^{L_{0}} d S\left[\frac{K}{2}\left(\frac{d^{2} Y}{d S^{2}}\right)^{2}-\frac{P}{2}\left(\frac{d Y}{d S}\right)^{2}\right],
$$

where $S$ is the arc length along the filament contour, $K$ is the bending rigidity, which relates the energy penalty of locally bending to the squared of local curvature ${ }^{6 / 9}$, $P$ is the applied force set to be positive for compression. The small-deflection assumption is used in the

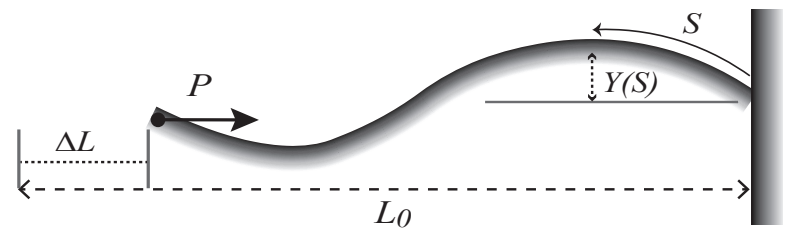

FIG. 1. A sketch of a filament under compression $P$, illustrating the notations used in the Euler model. above equation, giving $\frac{1}{2}(d Y / d S)^{2}$ as the local filament displacement along its average axis: the approach commonly adopted for semiflexible chains and vortex lines 10 . The important physical quantities to obtain here are: the force-displacement profile, which is the relation of how much the filament contracts upon increasing compressive forces, and the critical buckling force $P_{c}$, which is the threshold to initiate buckling and lose the mechanical stability ${ }^{5}$.

Based on the elastic Hamiltonian of Eq. (1), in 1757, Euler derived the relation for the critical buckling force, without thermal fluctuations ${ }^{5}$ : the classical expression is $P_{c}^{E}=\pi^{2} K / L_{0}^{2}$ for a filament pinned at both ends, where the numerical factor $\pi^{2}$ may change with different boundary conditions. One may also concider the role of thermal fluctuations that could affect the filament buckling 7111 . For a semi-flexible filament, thermal fluctuations cause the decrease of the critical buckling force, described as a simple scaling relationship11. $P_{c} / P_{c}^{E} \approx 1-1.11\left(k_{B} T L_{0} / K\right)^{0.56}$, where $K$ is the bending modulus as in Eq. (1). Thermal effects are less relevant for very stiff filaments, when $k_{B} T L_{0} / K \ll 1$. For a single MT of bending rigidity $K \approx 2 \times 10^{-23} \mathrm{~N} * \mathrm{~m}^{2}$ and length $L_{0}=10 \mu \mathrm{m}$ at room temperature, this ratio is estimated to be: $k_{B} T L_{0} / K \approx 0.002$. Consequently, thermal fluctuations have only a minor effect on MT buckling.

The Euler buckling model can only describe the single MTs in vitro, while the inclusion of an elastic matrix is more realistic in vivo, where the confinements of the CSK network could act as a source of elasticity. In fact, buckling experiments of single MTs, and the relating theories, have indicated that the embedding elastic matrix is an important factor. A larger critical buckling force $P_{c}$ and a shorter wavelength of the buckling pattern were observed in vivd 12 14, , compared with buckling without a matrix (in vitro). This difference in $P_{c}$ is qualitatively explained by the classical buckling theories on an 
inextensible filament without thermal fluctiations, due to Emil Winkler ca. 1866, which accounts for coupling with an elastic foundation that resists lateral displacement of the filament $5[14$. The Winkler elastic foundation model has the energy, $\frac{1}{2} \gamma Y^{2}$, added up to the Hamiltonian of Eq. (1), where $\gamma$ is the elastic modulus of the homogeneous embedding matrix.

For a long filament pinned at both ends, the Euler threshold $P_{c}^{E}$ decreases to zero, when the filament length $L_{0}$ becomes infinite. In contrast, Winkler obtains a length-independent $P_{c}$ relation that depends only on bending rigidity $K$ and the elastic modulus of the matrix: $P_{c}^{W}=2 \sqrt{K \gamma}$. Therefore, very long filaments are preferentially constrained by the matrix. Our work will focus on the details of the embedding elastic matrix, which both experiments and theory recognized as a crucial factor in MT buckling in vivo.

Although some theoretical papers have developed a more complicated descripton of the elastic matrix than used in the original Winkler model, the same buckling threshold is mostly recovered. An example is a non-linear elasticity model, with both transverse and longitudinal elastic coupling with the matrix ${ }^{15 / 16}$, aimed to explain the decay length of the buckling pattern: it seems that the Winkler expression for $P_{c}^{W}$ is very robust. However, we notice an unrealistic pre-assumption in these papers, and in the original Winkler model: the homogeneous elastic matrix. In cells, it implies the confinements of the network have a very small spacing, and the spatial variation of the elastic modulus along the filament is not allowed.

In reality, the intracellular cytoskeleton network ${ }^{17 / 18}$ can be rather disordered and inhomogeneous. This network is also highly dynamic ${ }^{19 \mid 20}$. The time-scales for the non-equilibrium soft matrix of cortical cytoskeleton to re-distribute its structure and reach thermal equilibrium are well-studied and are of the order of few minutes. This may be too long to be practically achieved when a microtubule finds itself under an external force, making this a quenched-disorder system. Quenched disorder refers to the 'frozen' heterogeneity that involves random variables which do not evolve with time and thermal fluctuations, due to its slow dynamics. Of course, there may be other in-vitro systems where the embedding matrix is disordered permanently. The effect of such disorder is conventionally formulated as a random potential that exhibits a characteristic probability distribution for each realization of the random variable. It has previously been seen to affect the average properties of polymers or filaments over all possible realizations, in many other different scenarios. For instance, the random potential acting on chain segments shrinks the mean size of the free polymer, inducing the collapse of the chain 21 23]; in filament stretching, the quenched random force exerted from the environment turns out to be an additional source of resistance to the stretching force applied on the filament ends, other than the non-zero temperature effect $24 \mid 25$. These studies motivate us to examine the effect of quenched disorder from the CSK confinements (reflected in the local variation of the elastic modulus) on the filament buckling.

The key findings of our paper are: the quenched disorder induces the softening of the matrix before and at buckling (decreasing the effective elastic modulus), and hence causes a decrease in the threshold $P_{c}$ from the Winkler expression. A critical point, similar to the switch between the first- and the second-order phase transition, appears in this effective elastic modulus and in the associated force-displacement profile, when the disorder strength increases. At buckling with weak disorder, an abrupt jump in the displacement appears, while a continuous change is seen in the strong disorder case. Although we find that thermal fluctuations are less relevant for MT in an inhomogeneous elastic matrix, the theory is capturing their effects, and hence its results remain valid even for longer or less stiff filaments, where thermal effects may become important.

The paper is organized as follows. Section 2.1 is on the the Hamiltonian of the system and the statistical distribution $f[\gamma]$ for the local realizations of the confining modulus $\gamma(S)$. In Section 2.2 we formulate the replica Hamiltonian $26, H_{\text {rep }}[Y(s)]$, required in finding the quench-averaged quantities. This Hamiltonian is not simple, and is Taylor-expanded to the 6th order of $Y$ : the 2nd order of this expansion is the elastic energy with an average elastic modulus, while the effect of strength of disorder is embedded in the negative 4 th and positive 6 th order terms. In Section 2.3. Gaussian variation method $(\mathrm{GVM})^{27 / 28}$ is introduced to deal with this high-order Hamiltonian. An optimization equation emerges from the requirement of varying the trial Gaussian Hamiltonian, in order to approach the true free energy of the system as closely as possible. In Section 3.1, we exclusively address the optimization equation in the long filament. In Section 3.2, based on this equation, the trial Gaussian is solve as a function of the compressive force and the strength of disorder. Sections 3.2 to 3.5 discuss the consequences of the solved trial function to obtain $P_{c}$ and $\langle\Delta L\rangle$. To keep the text neat and the logical line clear, many of the detailed derivations are moved to the Appendices.

\section{THEORY}

\subsection{Hamiltonian and quenched disorder distribution}

We consider MT as an inextensible filament of length $L_{0}$, embedded in an inhomogeneous elastic matrix of the $2 \mathrm{D}$ system. The regime before and at buckling is the main interest of this paper, hence imposing the the small-deflection assumption $5|10| 15|16| 24$ to avoid the complexity that could arise in the elastica theory $\sqrt{5}$. As a simple model, the matrix is assumed to show linear elasticity only in the lateral direction, with the local elastic modulus $\gamma(S)$, which embodies the nature of randomlydistributed confinements along the filament. The reason 
(A)

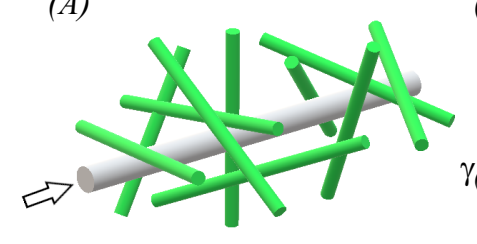

(B)

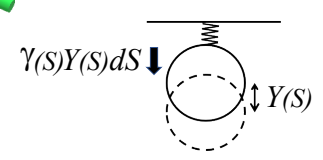

FIG. 2. (A) A filament laterally constrained by randomlydistributed confinements along the axis, imposing local sites of elastic force penalizing the transverse filament displacement. (B) The cross-section viewed from the arrow. An effective spring of elastic constant $\gamma(S) d S$ is used to determine this confining force: $\gamma(S) Y(S) d S$, for the filament segment of length $d S$.

to exclude longitudinal elasticity is because it showed no qualitative difference in critical buckling force in earlier studies $15 \mid 16$.

Each realization of the local elastic modulus $\gamma(S)$ throughout the filament contour occurs with the probability given by $f[\gamma(S)]$. Despite inhomogeneity, the elasticity strength of this matrix is measured by its average elastic modulus $\langle\gamma\rangle$. The symbol $\langle\cdots\rangle$ means averaging over all possible realizations of $\gamma(S)$ along the filament contour, through its probability distribution function $f[\gamma]$. Note that our model is reminiscent of Winkler's, yet the local elastic modulus here is no longer a constant. The goal is to use the well-developed replica technique $e^{26 \mid 27}$ to find how strength of quenched disorder enters the critical buckling force $\left\langle P_{c}\right\rangle$ and forcedisplacement $\langle\Delta L\rangle$.

The Hamiltonian of the system described in Fig. 2 is modified from Eq. (1), by adding an elastic energy stored in the matrix:

$$
H=\int_{0}^{L_{0}} d S\left[\frac{K}{2}\left(\frac{d^{2} Y}{d S^{2}}\right)^{2}-\frac{P}{2}\left(\frac{d Y}{d S}\right)^{2}+\frac{\gamma(S)}{2} Y^{2}\right]
$$

where the local elastic modulus $\gamma(S)$ is the quenched disordered variable. To find its statistical distribution $f\left[\gamma(S], w\right.$ segments of length $\Delta_{S}$ along the filament are cut from the matrix: $w=L_{0} / \Delta_{S}$. Within each $\Delta_{S}$ segment, the elastic modulus for this local segment is assigned the notation $\gamma_{i}$. The same cutting-counting procedure is repeated numerous times for many MT filaments to build up the probability distribution function of the number of confinements $N_{i}^{c}$, for this local segment of length $\Delta_{S}$. Here, the confinements refer to the filaments that are parts of the intracellular network and have contact with the main filament we are investigating on the surface (see Fig. 2).

This distribution function of $N_{i}^{c}$, however, is not available from previous studies. Yet, it may be tentatively assumed to follow the Poisson distribution, which adopts a constant average rate for occurrence of individual events. In the context here, it means that, on average, it is expected to find one confinement when moving along the

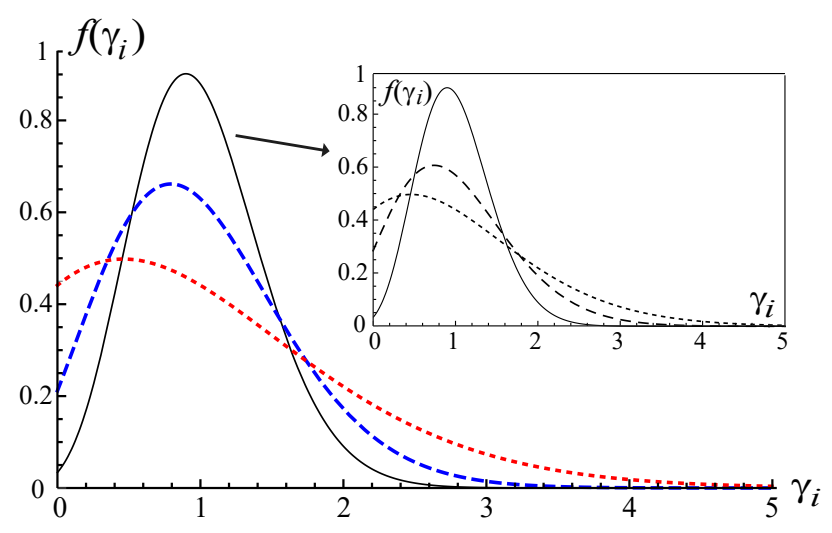

FIG. 3. The distribution function $f\left(\gamma_{i}\right)$, Eq. (3), which depends on the ratio $\left(\gamma \Delta_{S} / \varepsilon\right)$, is plotted at fixed $\langle\gamma\rangle=1$ and $\Delta_{S}=5$. A broadening of the distribution width is observed when the elastic strength of confinement $\varepsilon$ is increased: $\varepsilon=1$ (solid line), $\varepsilon=2$ (dashed line), and $\varepsilon=5$ (dotted line). The inset shows $f\left(\gamma_{i}\right)$ at fixed $\varepsilon=5$ and varying segment length: $\Delta_{S}=5$ (solid), $\Delta_{S}=3$ (dashed), and $\Delta_{S}=1$ (dotted line).

filament contour across a distance $\xi$, the average spacing between CSK confinements. In fact, the limiting expression of Poisson (when the segment length $\Delta_{S}$ is long enough, it roughly gives a Gaussian with the mean and variance equal to the same value) was already used to formulate the probability distribution of the density of the quuenched cross-links 28 .

Here, we write the general form of the Poisson distribution with respect to $N_{i}^{c}$ as: $\left(\Delta_{S} / \xi\right)^{N_{i}^{c}} \exp \left(-\Delta_{S} / \xi\right) / N_{i}^{c}$ !, where $\Delta_{S}$ shall be multiples of $\xi$. We can convert this probability of $N_{i}^{c}$ into the probability distribution of $\gamma_{i}$, through the relation $\gamma_{i} \Delta_{S}=N_{i}^{c} \varepsilon$, where $\varepsilon$ is the elastic constant a single confinement provides, and the average elastic modulus is given as $\langle\gamma\rangle=\varepsilon / \xi$. Note that $\xi$ and $\langle\gamma\rangle$ are parameters that can be experimentally measured. The direct measurement of $\varepsilon$ is not plausible, but its value can be deduced from the relation $\varepsilon=\langle\gamma\rangle \xi$. Therefore, the Poisson distribution we use here can build a connection with the experiments. The elastic constant $\varepsilon$ depends on many factors that are not yet specified, such as the orientation or the bending rigidity of the confinements. It is also possible that $\varepsilon$ has an entropic excludedvolume contribution proportional to $T$. However, we do not intend to complicate the problem further, and take the parameter $\varepsilon$ as a somewhat averaged value, so as to see more easily the effect of quenched disorder (i.e. the local distribution of the number of confinements along the filament).

The probability distribution $f[\gamma]$ for a $\gamma(S)$ realization throughout the whole filament is simply the product of the probability for each segment:

$$
f[\gamma]=\frac{1}{\mathcal{N}} \prod_{i=1}^{w} \frac{\left(\frac{\Delta_{S}\langle\gamma\rangle}{\varepsilon}\right)^{\gamma_{i} \Delta_{S} / \xi} e^{-\Delta_{S}\langle\gamma\rangle / \varepsilon}}{\left(\gamma_{i} \Delta_{S} / \varepsilon\right) !},
$$

where $\mathcal{N}$ is the normalization factor, the mean elastic 
modulus $\langle\gamma\rangle=\varepsilon / \xi$. Based on this distribution, the variance $\left(\left\langle\Delta \gamma_{i}^{2}\right\rangle-\left\langle\Delta \gamma_{i}\right\rangle^{2}\right)$ in the segment of length $\Delta_{S}$ is $\varepsilon^{2} / \Delta_{S} \xi$ (or equally: $\langle\gamma\rangle \varepsilon / \Delta_{S}$ ). For matrices sharing the same average elastic modulus, the choice of $\varepsilon$ will affect the disorder strength affecting the local elastic modulus $\gamma(S)$. The matrix with a larger $\varepsilon$, or equally a larger spacing $\xi$ as we hold $\langle\gamma\rangle=\varepsilon / \xi$ at a fixed value, has a broader distribution width of $\gamma_{i}$ and thus stronger disorder, if compared in segments of the same length $\Delta_{S}$. In Fig. 3, we present the width-broadening feature when increasing the disorder measure $\varepsilon$ or decreasing the segment length $\Delta_{S}$, while keeping the same mean elastic modulus $\langle\gamma\rangle$.

When we use the notation $\int D \gamma f[\gamma]$ for the path integral over all possible realizations of $\gamma(S)$ along the filament, it means to discretize the filament in segments of length $\Delta_{S}$ first, and then complete the averaging. The quenched disorder free energy $F_{d}$ of this system is given by: $F_{d}=-k_{B} T\langle\ln Z\rangle=k_{B} T \int D \gamma f[\gamma] \ln Z$, where $Z$ is the partition function obtained by integrating over configurations of $Y$, namely, $Z=\int D Y \exp (-\beta H)$ (the expression of $H$ is given in Eq. (2)), and $\beta=$ $1 / k_{B} T$. Likewise, given a physical quantity ' $A$ ' which depends on the specific choice of the $\gamma(S)$ realization, its quenched average $\langle A\rangle$ is estimated by: $\langle A\rangle=$ $\int D \gamma f[\gamma] \int D Y\left(A e^{-\beta H} / Z\right)$, with the same definition of $Z$ above. Although simple and clear in concept, the direct implementation of this quenched averaging is algebraically difficult, which, nevertheless, can be overcome by the replica technique 2126 .

\subsection{Replica Technique}

Replica technique was invented by Edwards and Anderson in $1976^{29}$, to study the spin-glass system, and then widely applied to other quenched disordered systems $2123|24| 30$. The idea is to re-write $\ln Z$ as a limit expression, which facilitates the averaging process by quenched disorder 26131: $F_{d}=-k_{B} T\langle\ln Z\rangle=$ $-\left.k_{B} T\left(\partial\left\langle Z^{m}\right\rangle / \partial m\right)\right|_{m \rightarrow 0}$. The first step is to calculate the averaged replica partition function $\left\langle Z^{m}\right\rangle$, with an integer $m$. This is physically equivalent to averaging the disorder across $m$ replicas of the investigated system with the same Hamiltonian, hence the name. A bald step is then taken: sending the number of replicas $m$ continuously to zero while the averaging $\left\langle Z^{m}\right\rangle$ is done assuming $m$ to be an integer.

Both the disorder free energy and any average $\langle A\rangle$ are usually given through the replica Hamiltonian, $H_{\text {rep }}$. Its definition is simply the quenched averaged Hamiltonian of all $m$ replicas, and is the exponent remained after carrying out the $\gamma(S)$ path integral inside $\left\langle Z^{m}\right\rangle$ (without further integration over configurations of the filament deflection), through the relation:

$$
e^{-\beta H_{\mathrm{rep}}} \equiv \int D \gamma f[\gamma] e^{-\beta \sum_{a=1}^{m} H\left[Y_{a}, \gamma\right]},
$$

where $H\left[Y_{a}, \gamma\right]$ is the Hamiltonian of the ath replica: $H_{a}=\int_{0}^{L_{0}} d S\left(K Y_{a}^{\prime \prime 2} / 2-P Y_{a}^{\prime 2} / 2+\gamma(S) Y_{a}^{2} / 2\right)$ from Eq. (2). $Y_{a}(S)$ is the filament deflection in the ath replica, and the shorthand ' and " refer to differentiation with respect to the coordinate $S$ once and twice, respectively. The quenched averaged quantities are expressed through $H_{\text {rep }}$ as:

$$
\langle A\rangle=\lim _{m \rightarrow 0} \int\left(\prod_{a=1}^{m} D Y_{a}\right) A_{\{a, b\}=1} e^{-\beta H_{\mathrm{rep}}} .
$$

The label ' 1 ' used for the replica of the observable ' $A$ ' is completely arbitrary, and in fact, the result obtained must not depend on this particular index if the calculation is done correctly.

The calculation of $H_{\text {rep }}$ is straightforward 2426 . The energy terms without $\gamma(S)$ in Eq. (4) are unchanged after completing the path integral over $\gamma(S)$. For terms containing $\gamma(S)$, the standard procedure to carry out this path integral involves a similar discretization process of $S$ and $\gamma(S)$, as described in Section 2.1 cutting each of the total $m$ replicated filaments into $w$ segments of length $\Delta_{S}, w=L_{0} / \Delta_{S}$. But this time $Y_{a}(S)$ is also involved in the path integral, and also needs to be discretized. It is implicitly assumed that the continuous filament deflection functions $Y_{a}$ in all replicas are smooth and slowlyvarying, which is usually true before the buckling occurs. The continuous function $Y_{a}(S)$ could therefore be reasonably replaced with its discretized version $Y_{a, i}$, the filament deflection of the $i$ th segment in the $a$ th replica.

With the discretized functions $Y_{a, i}$ and $\gamma_{i}$, and the $f[\gamma]$ from Eq. (3), the terms involving $\gamma(S)$ can be integrated in the path integral in Eq. (4), with the use of the Stirling approximation for the factorial and then by finding out the steepest descent of $f\left(\gamma_{i}\right) \cdot \exp \left(-\gamma_{i} \sum_{a} Y_{a, i}^{2} \Delta_{S} / 2\right)$ (see Appendix A). We Taylor-expanded this resultant function in the assumption of small deflections of $Y_{a, i}^{2}$, which was already adopted in formulating the Hamiltonian in Eq. (2). The expansion has to go to the 6 th order term, since we discover that the 4 th order term is negative and does not provide system stability. Subsequently we transform the discretized version back to the continuous integral with the approximated $H_{\text {rep }}$ written as:

$$
\begin{gathered}
H_{\text {rep }}=\int_{0}^{L_{0}} d S \sum_{a=1}^{m}\left(\frac{K}{2} Y_{a}^{\prime \prime 2}-\frac{P}{2} Y_{a}^{\prime 2}+\frac{\langle\gamma\rangle}{2} Y_{a}^{2}\right) \\
-\sum_{a, b=1}^{m} \frac{\beta\langle\gamma\rangle \varepsilon}{8} Y_{a}^{2} Y_{b}^{2}+\sum_{a, b, c=1}^{m} \frac{\beta^{2}\langle\gamma\rangle \varepsilon^{2}}{48} Y_{a}^{2} Y_{b}^{2} Y_{c}^{2},
\end{gathered}
$$

where $K$ is the bending rigidity, $P$ the compressive force, $\varepsilon$ the elastic constant of a single confinement, and $Y_{a}$ the filament deflection along the filament contour $S$ of the ath replica.

The disorder strength in the local confinements of our filament are measured by the distribution width: $\left\langle\Delta \gamma_{i}^{2}\right\rangle-\left\langle\Delta \gamma_{i}\right\rangle^{2}=\langle\gamma\rangle \varepsilon / \Delta_{S}$. Only the 4th and 6th 
order terms reflect this disorder effect from confinements through the parameter $\varepsilon$, while the harmonic term $\langle\gamma\rangle Y_{a}^{2} / 2$ represents the Winkler limit of homogeneous elastic foundation. This can be understood from the fact that to hold a constant mean elastic modulus, a weaker elastic strength $\varepsilon$ from a single confinement requires a shorter spacing of confinements (higher confinement density), eventually giving the limiting homogeneous case. On the other hand, when there is no confinement, the mean elastic modulus $\langle\gamma\rangle$ becomes zero, causing the Winkler and higher-order terms to vanish, and the Euler limit is recovered.

A similar problem was considered earlier ${ }^{32}$, and only retained to the 4 th-order term of the polymer field, also with a negative coefficient. There, the authors introduced the auxiliary field to decouple the 4th-order term, then expanded the resultant Hamiltonian into many harmonic terms, and recognized the divergent term as the main contribution to the 'propagator'. Nevertheless, the physical quantity they were looking for had later to be normalized by all the contributions in this propagator, and thus were able to deal with the resulting instability (divergence). However, this method would not work in our model, as the investigated physical quantities are totally different. We don't have the same normalization process to counter this divergence, and consequently need the 6 th order term here to avoid instability.

To find the quenched averaged quantities, we need to insert this $H_{\text {rep }}$ back into Eq. (5). We introduce the Gaussian variation method (GVM) for high-order terms, which was first used in the context of random manifolds by Mezard and Parisi 27 and widely applied in quenched disorder systems 23128 . In particular, our $H_{\text {rep }}$ takes a very similar structure to the problem of a free polymer chain in random potential23. Before implementing the GVM, we non-dimensionalize Eq. (6) and re-write it in the new non-dimensional variable $s=S / \zeta$ and deflection $y_{a}=Y_{a} / \zeta$, where $\zeta$ is an arbitrary length scale, which we expect to be the order of MT segment size. We will need to later verify that none of our results depend on the choice of this length scale. We need this parametrization (instead of choosing the length scale to be the filament length $L_{0}$ ) in order to leave the actual MT length $L_{0}$ free to change and also recognize the major contributions in the free energy for a long filament, as some results will depend on it.

$$
\begin{aligned}
\tilde{H}_{\text {rep }} & =\int_{0}^{l_{0}} d s \sum_{a=1}^{m}\left(\frac{k}{2} y_{a}^{\prime \prime 2}-\frac{p}{2} y_{a}^{\prime 2}+\frac{æ}{2} y_{a}^{2}\right) \\
& -\sum_{a, b=1}^{m} \frac{\eta}{4} y_{a}^{2} y_{b}^{2}+\sum_{a, b, c=1}^{m} \frac{w}{6} y_{a}^{2} y_{b}^{2} y_{c}^{2} \\
\text { with } \eta & =\frac{æ \Delta}{2} \text { and } w=\frac{æ \Delta^{2}}{8},
\end{aligned}
$$

where $l_{0}, \tilde{H}_{\text {rep }}, k, p, æ$ and $\Delta$ are non-dimensional forms defined by the relations: $l_{0}=L_{0} / \zeta, \tilde{H}_{\text {rep }}=\beta H_{\text {rep }}$, $k=\beta K / \zeta, p=\beta P \zeta, æ=\beta \zeta^{3}\langle\gamma\rangle$ and $\Delta=\beta \zeta^{2} \varepsilon$. The non-dimensional mesh size $l_{m}$ is accordingly expressed as: $l_{m}=\xi / \zeta=\Delta / æ$. $y_{a}^{\prime \prime}$ and $y_{a}^{\prime}$ are the second and first derivatives of the filament deflection of the $a$ th replica, with respect to the non-dimensional contour length $s$. Note that the coefficients $\eta$ and $w$ are not independent, and in the final results, they will be transformed back to $æ$ and $\Delta$ parameters. Eq. (7) is the key result in this section and is the non-dimensional replica Hamiltonian that will be used in the following calculations.

\subsection{GVM and the optimization equation}

The standard GVM procedures go as follows. The replica Hamiltonian is first cast into Fourier space, to handle any differential terms of the fields $y_{a}$ in Eq. (7). We use the boundary conditions of a filament pinned at both ends $\sqrt{14}$, requiring $d y_{a} / d s=0$ and $d y_{a}^{2} / d s^{2}=0$. The $y_{a}$ is accordingly written as a sine Fourier series: $y_{a}=\sum_{n=1}^{\infty} \bar{y}_{a n} \sin \left(n \pi s / l_{0}\right)$, where ' $n$ ' is the index for the discrete Fourier modes, $\bar{y}_{a n}$ is the Fourier amplitude of the $n$-th mode in the ath replica. Inserting this series back in Eq. (7), and integrating over $s$, we obtain:

$$
\begin{aligned}
\tilde{H}_{\text {rep }}= & \sum_{a=1}^{m} \sum_{n}^{\infty} \frac{l_{0}}{4}\left[k\left(\frac{n \pi}{l_{0}}\right)^{4}-p\left(\frac{n \pi}{l_{0}}\right)^{2}+æ\right] \bar{y}_{a n}^{2} \\
& -\frac{\eta l_{0}}{32} \sum_{a, b} \sum_{\{n\}} \Gamma_{4} \bar{y}_{a n_{1}} \bar{y}_{a n_{2}} \bar{y}_{b n_{3}} \bar{y}_{b n_{4}} \\
+ & \frac{w l_{0}}{192} \sum_{a, b, c} \sum_{\{n\}} \Gamma_{6} \bar{y}_{a n_{1}} \bar{y}_{a n_{2}} \bar{y}_{b n_{3}} \bar{y}_{b n_{4}} \bar{y}_{c n_{5}} \bar{y}_{c n_{6}},
\end{aligned}
$$

where $\sum_{\{n\}}$ means summing over all Fourier mode indices, the suffixes $a$ and $b$ are replica indexes (summed to the number of total replicas, $m$ ), and $\Gamma_{4}$ and $\Gamma_{6}$ are assemblies of many Kronecker-deltas of different Fourier modes (e.g. one of the terms inside $\Gamma_{4}$ is $\delta\left(n_{1}+n_{2}-\right.$ $\left.n_{3}-n_{4}\right)$ ), produced due to the integral of multiple sinfunctions. The full expressions of $\Gamma_{4}$ and $\Gamma_{6}$ are not given here, due to its long length. Instead, the $\Gamma_{4}$ case (i.e. the 4th order case) will be put in Appendix B, for the illustration of algebraic manipulation.

A trial quadratic Hamiltonian in the $\bar{y}_{a n}$ functions is assumed to describe the system, which means all physical properties, such as free energy and the quench-averaged quantities, are defined by this new Hamiltonian $\tilde{H}_{0}$, instead of the original high-order Hamiltonian $\tilde{H}_{0}$. Although Eq. (8) contains interactions between different Fourier modes, the trial Hamiltonian is assumed to include only the interactions within the same modes. It seems a primary intention of approximation, yet has been shown to very likely capture the key physical essence underlining the problem 23 . It is also important that this trial function does incorporate the interactions between different replicas, which appeared as high-order contributions in our original $\tilde{H}_{\text {rep }}$ and are, in fact, crucial and frequently discussed on their play in the replica 
theory $26|27| 30 \mid 31$. The replica symmetry assumption ${ }^{26}$ is further applied, allowing the simplification that only two variational parameters are needed; its stability in this kind of problems has been verified earlier ${ }^{23}$. A more complicated replica symmetry-breaking case will not be presented in this paper. The structure of the trial Hamiltonian is consequently given as:

$$
\begin{aligned}
\tilde{H}_{0} & =\frac{1}{2} \sum_{a, b}^{m} \sum_{n} \bar{y}_{a n} G_{a b}^{-1}(n) \bar{y}_{b n} ; \\
G_{a b}^{-1}(n) & =l_{0}\left\{\Lambda(n) \delta_{a b}+\sigma(n) I_{a b}\right\},
\end{aligned}
$$

where $G_{a b}^{-1}(n)$ is the element of an $(m \times m)$-matrix at the $n$-th Fourier mode, with the suffixes $a$ and $b$ for the replica indexes, and $m$ for the total replica number. As often in this type of analysis, $I_{a b}$ is the $(m \times m)$ matrix of ones. Since we see a $l_{0}$ factor from all contributions in the replica Hamiltonian of Eq. (7), we expect this $l_{0}$ can also be extracted in our trail Hamiltonian. The variational parameters are $\Lambda$ and $\sigma$, and their $n$-dependence, will be revealed later, when we find out the optimization equation in the GVM, which we are about to address.

The free energy of the original system described by $\tilde{H}_{\text {rep }}$ can be cast as an expansion of $\left\langle\tilde{H}_{\text {rep }}-\tilde{H}_{0}\right\rangle_{0}$ :

$$
\tilde{F} \approx \tilde{F}_{0}+\left\langle\tilde{H}_{\text {rep }}-\tilde{H}_{0}\right\rangle_{0},
$$

where $\tilde{F}_{0}$ is the free energy using the trial Hamiltonian and has the relation: $\tilde{F}_{0}=-\sum_{n} \operatorname{Tr} \ln \underline{\underline{G}}(n) / 2$, and the symbol $\langle\cdots\rangle_{0}$ means averaging the quantity by the partition of the new trial Hamiltonian $\exp \left(-\tilde{H}_{0}\right)$. The aim of GVM is to minimize Eq. (10) with respect to the variational parameters $\Lambda$ and $\sigma$. The calculation of $\left\langle\tilde{H}_{\text {rep }}-\tilde{H}_{0}\right\rangle_{0}$ in Eq. $(10)$ is straightforward with the help of the Wick theorem ${ }^{35}$, yet lengthy, where the pairing of Fourier modes needs discussion, so as to eliminate the Kronecker deltas in $\Gamma_{4}$ and $\Gamma_{6}$. The full expression of $\tilde{F}$ is derived in Appendix. B, and in the case of a long filament, $\tilde{F}$ is approximately given by:

$$
\begin{gathered}
\tilde{F} \approx-\frac{1}{2} \sum_{n} \operatorname{Tr} \ln \underline{\underline{G}}(n)+\sum_{a} \sum_{n_{1}} \frac{l_{0} \psi(1)}{2} G_{a a}^{(1)} \\
-\frac{\eta l_{0}}{16} \sum_{a, b} \sum_{\{n\}}\left(G_{a a}^{(1)} G_{b b}^{(2)}+2 G_{a b}^{(1)} G_{a b}^{(2)}\right) \\
+\frac{w l_{0}}{48} \sum_{a, b, c} \sum_{\{n\}}\left(G_{a a}^{(1)} G_{b b}^{(2)} G_{c c}^{(3)}+6 G_{a a}^{(1)} G_{b c}^{(2)} G_{b c}^{(3)}\right. \\
\left.+8 G_{a b}^{(1)} G_{a c}^{(2)} G_{b c}^{(3)}\right),
\end{gathered}
$$

where we move the Fourier mode index of $G_{a b}(n)$ to the superscript by using the shorthand of ' 1 ' to ' 3 ' for $n_{1}$ to $n_{3}$, and $\psi(1)=\left(k\left(n_{1} \pi / l_{0}\right)^{4}-p\left(n_{1} \pi / l_{0}\right)^{2}+æ\right) / 2$. The second and third lines of Eq. (11) has the same structure as in Ref[23, where a similar math procedure was carried out for free polymers (i.e. without the pinning boundary conditions used here, and hence with continuous Fourier bases). On the other side, for a short-filament, this variational free energy $\tilde{F}$ will have a lot more terms similar in nature to the ones given above. The discarded terms in the full $\tilde{F}$ expression are very likely the contributions from the boundary conditions, and become important in the short-filament case.

In the long-filament case, we can follow the Mézard and Parisi steps $\$ 23 / 27 / 28$, and write $\Lambda(n)$ in the form: $\Lambda=\vartheta(n)+\lambda$, where $\vartheta$ is chosen to match the quadratic contributions of Eq. (8). The detailed derivation is given in Appendix C. Overall, we write:

$$
\begin{aligned}
G_{a b}^{-1}(n) & =l_{0}\left\{[\vartheta(n)+\lambda] \delta_{a b}+\sigma I_{a b}\right\} \\
\text { with } \vartheta(n) & =\frac{1}{2}\left[k\left(\frac{n \pi}{l_{0}}\right)^{4}-p\left(\frac{n \pi}{l_{0}}\right)^{2}+æ\right],
\end{aligned}
$$

where the parameters $\lambda$ and $\sigma$ are used to account for the quenched disorder effects from the 4th- and 6th-order terms in Eq. 8). In particular, the off-diagonal effect (interactions between two different replicas) is imposed through $\sigma$. Noticeably, $\lambda$ and $\sigma$ are not $n$-dependent, and all $n$-dependence is embedded into $\vartheta(n)$.

The kernel $\vartheta(n)$ of Eq. (12) was also used in the criterion for the critical force in the Winkler model, where the parameters $\lambda$ and $\sigma$ accounting for the disorder effect are set to zero in the correlator $G_{a b}^{-1}(n)$. When the compression $p$ is increased and eventually $G_{a b}^{-1}$ is allowed to have a negative value (expecting the divergence in the thermal partition), the buckling occurs. This is equivalent to finding the condition for $p$ that makes the equation $\vartheta(n)=0$ first have a real solution. When $\left(n \pi / l_{0}\right)$ is treated as a continuous wave vector, we recover the Winkler result: $p_{c}=2 \sqrt{k æ}$ in the long filament case.

On the other hand, in the short-filament case, the use of the full expression of $\tilde{F}$ (Appendix. B) will change the $n$-dependence of $\Lambda$ in Eq. (9). For example, in the simplified case when only the 4th-order Hamiltonian is used in Eq. (7), $\Lambda(n)$ takes the form $[\psi+\lambda+$ $\left.\sqrt{(\psi+\lambda)^{2}-\eta / 2 l_{0}}\right] / 2$, where $\lambda$ is an $n$-independent variational parameter that shall further be solved, while $\psi$ is the source of the $n$-dependence defined below Eq. (11). This derivation is given in Appendix C. For the 6th-order case of a short filament, finding $n$-dependence becomes algebraically implausible, and prevents us from any further analysis of the variational parameters.

We will choose to stay in the long-filament regime, not just due to the complexity the short-filament regime has, but also because earlier experiments have shown that the Winkler model in the long filament regime, rather than in the short-filament case, could provide a reasonable explanation for $p_{c}$ and buckling wavelength in the intracellular environments 14 . The short-filament case will hence not be addressed in this paper.

The optimization of $\lambda$ and $\sigma$ in Eq. (12) is fulfilled by finding the minimum of $F$ in Eq. (11), through the functional derivative $\delta F / \delta G_{a b}(n)=0$. Generally speaking, $\sigma$ and $\lambda$ in Eq. (12) are obtained as functions of the total number of replicas $m$. The optimized trial 
Hamiltonian $\tilde{H}_{0}$, with the solved $\lambda$ and $\sigma$ functions inside, are subsequently placed back in Eq. (5) in the nondimensional form and Fourier space, then taking the limit $m \rightarrow 0$. It turns out that this standard procedure are algebraically implausible, particularly in solving the optimization equation and expressing $\sigma$ and $\lambda$ in functions of $m$.

In fact, if the replica number $m$ is sent to zero, at this step of deriving the optimization equation, the consequent equation is greatly facilitated and becomes mathematically manageable. The solutions obtained following this simplification are $\lambda_{0}$ and $\sigma_{0}$, i.e. taking $\lambda$ and $\sigma$ to the limit $m \rightarrow 0$, which are the only quantities needed in estimating the quench-averaged values $\langle A\rangle$. Therefore, we use the limit $m \rightarrow 0$ here for the equation $\delta F / \delta G_{a b}(n)=0$ and obtain:

$$
\begin{aligned}
G_{a b}^{-1}(n) & \approx l_{0} \vartheta(n) \delta_{a b} \\
& +\frac{l_{0}}{2}\left(-\eta \sum_{n_{1}} G_{a b}^{(1)}+w \sum_{i}^{m} \sum_{\{n\}} G_{a i}^{(1)} G_{i b}^{(2)}\right) I_{a b} .
\end{aligned}
$$

Note that the term $\sum_{i}^{m} \sum_{\{n\}} G_{a i}^{(1)} G_{i b}^{(2)}$ still has $m$ dependence buried inside. To remove this $m$-dependence, the structure of $G_{a b}$ is required. The inverse $G_{a b}^{-1}$ in Eq. (12) can easily be transformed to give $G_{a b}$ as:

$$
G_{a b}(n)=\frac{1}{l_{0}(\vartheta+\lambda)}\left[\delta_{a b}-\frac{\sigma}{(\vartheta+\lambda+m \sigma)} I_{a b}\right] .
$$

Inserting Eqs. (12) and (14) into Eq. (13) and comparing the off-diagonal and diagonal elements gives two equations of $\sigma$ and $\lambda$, under the limit $m=0$. The first equation takes the form:

$$
\lambda_{0}=-\frac{\eta}{2 l_{0}}\left(\sum_{n} \frac{1}{\vartheta+\lambda_{0}}\right)+\frac{w}{2 l_{0}^{2}}\left(\sum_{n} \frac{1}{\vartheta+\lambda_{0}}\right)^{2},
$$

where the subscript of $\lambda_{0}$ indicates that this is the $\lambda$ solution in the limit $m=0$. Since the right hand side of Eq. 15 has summing up of the $n$-dependence in $\vartheta$, $\lambda_{0}$ does not depend on ' $n$ '. We remind again that when there is no disorder (i.e. $\eta=w=0$ ), this equation gives $\lambda=0$, as expected in the Winkler model.

The second optimization equation would give $\sigma_{0} \cdot \varphi=0$, where $\varphi$ is a function of $p, k, æ, l_{0}$ and $\lambda_{0}$. If $\sigma_{0}$ is arbitrary, the function $\varphi$ is required to be zero, which accordingly generates another equation for $\lambda_{0}$. It is unlikely that this equation would match Eq. (15). To allow a possible solution for $\lambda_{0}, p$ (the only adjustable parameter) will have to be a specific value, which is unlikely to still be physically reasonable (e.g. $p$ may be required to be a complex number). Instead, a more practical and rather trivial solution is adopted: $\sigma_{0}=0$, making the correlator $G_{a b}^{-1}$ of the trial Hamiltonian a diagonal matrix. Note that this diagonal form of the trial Hamiltonian is not uncommon, and has first emerged in the EdwardsMuthukumar work 21 .

\section{RESULTS AND DISCUSSION}

\subsection{The limit of long filaments}

In Section 2.3, we elected to stay at the long-filament regime and give the expression of $\vartheta(n)$ in Eq. (12). In this regime, the sum $\sum_{n}$ in Eq. (15) can be replaced with the continuous integral $\int d n$. We set the wavenumber $q=n \pi / l_{0}$, therein $\int d n \rightarrow l_{0} \int d q / \pi$, with the lower limit of the $q$-integral being $\pi / l_{0}$ (the upper limit remains infinite, in what we assume to be a continuous filament). From the term $\vartheta+\lambda_{0}$ in Eq. (12), the disorder effect is incorporated with the averaged elastic modulus $æ$, giving the effective elastic modulus $\alpha: \alpha=æ+2 \lambda_{0}$. We will also replace $\lambda_{0}$ with this effective elastic modulus $\alpha$. Equation 15 is re-written in $q$ and $\alpha$ as:

$$
\begin{aligned}
\alpha & =æ-\eta B+w B^{2} ; \\
\text { with } B & =\frac{2}{\pi} \int_{\pi / l_{0}}^{\infty} \frac{d q}{k q^{4}-p q^{2}+\alpha},
\end{aligned}
$$

where $\alpha$ is the effective elastic modulus defined as: $æ+2 \lambda_{0}$. The $B$-integral above exhibits two different types of divergence behavior when plotted against $p$, depending on the value of the lower integral limit $\pi / l_{0}$ (in other words, for long and short filaments). This can be observed from the denominator inside the integral: $\left(k q^{4}-p q^{2}+\alpha\right)$. The divergence occurs when the $q$ value in the integrating range makes this denominator zero.

We gradually increase $p$ from zero, and define $p^{*}$ as the value that can first have this denominator diminish to zero at a specific wavenumber $q^{*}$. These two values $p^{*}$ and $q^{*}$ are easily found out from the quadratic $k q^{4}-$ $p q^{2}+\alpha=0$, and given as:

$$
p^{*}=2 \sqrt{k \alpha} ; q^{*}=\sqrt[4]{\alpha / k} .
$$

If the lower integral limit $\pi / l_{0}$ is smaller than $q^{*}$ at $p=p^{*}$, the $B$-integral diverges. This $p^{*}$ expression is reminiscent of the critical buckling force $p_{c}$ for a long filament in the Winkler model, but here we have the effective elastic modulus $\alpha$ that contains the disorder effect, instead of the average elastic modulus æ without this effect. On the other hand, if $\left(\pi / l_{0}\right)>q^{*}$ at $p=p^{*}$ (in the short-filament regime), the $B$-integral will not diverge, until the $q$ solution of the equation, $k q^{4}-p q^{2}+\alpha=0$, eventually overlaps with the lower limit $\pi / l_{0}$, when one further increases $p$ beyond $p^{*}$. This will give the divergence that depends on the filament length $l_{0}$. We will not further discuss this interesting result, and only focus on the long-filament regime, which the expression of our correlator $G_{a b}^{-1}$ is based in.

Here we obtained a more concrete condition to identify the long-filament regime: $l_{0}>\pi(k / \alpha)^{1 / 4}$, and also realize that the $B$-integral diverges at $p=2 \sqrt{k \alpha}$. For a really long filament $\left(l_{0} \rightarrow \infty\right)$, the $B$-integral will take 
the limiting form:

$$
B=\frac{1}{\sqrt{\alpha}(2 \sqrt{k \alpha}-p)^{1 / 2}},
$$

which only covers the regime $2 \sqrt{k \alpha}-p>0$ before divergence occurs, while the regime $2 \sqrt{k \alpha}-p<0$ has no real physical meaning.

To include the effect of the finite filament length in this regime, one has to add extra terms in series expeansion of $1 / l_{0}$, but such a correction is not necessary for the case of MT inside the cell. We choose the length scale $\zeta$ to be $10 \mathrm{~nm}$ (roughly the size of the tubulin dimers 34 , which are the basic units composing MT filaments), then insert reasonable values of the parameters $k$ (with the bending rigidity ${ }^{12[35 \mid 36} K$ between $10^{-23}$ and $10^{-24} \mathrm{~N} \mathrm{~m}^{2}$ ) and the $\alpha$ values between $10^{-1}$ and 10 (covering the experimentally-determined elastic modulus of the cell matrix, of a few $\mathrm{kPa}(17 / 37 / 38)$, and compare the numerical results with Eq. (18). The long filament turns out to be a good approximation for an MT of length above $3 \mu \mathrm{m}$ (the range of the MT length commonly seen in in-vivo experiments14). We hence use Eq. (18) for the remaining calculations: to solve Eq. (16) with Eq. (18) for $B(\alpha, p)$, insert $\lambda_{0}$ and $\sigma_{0}$ back into $H_{0}$ of Eq. (9), and replace $\tilde{H}_{\text {rep }}$ with $\tilde{H}_{0}$ in calculating Eq. (5).

\subsection{Solution path of the effective elastic modulus $\alpha$}

In order to investigate how the increase of the compressive force $p$ affects the quenched averaged quantities of the system, the optimization Eq. (16) should solved for $\alpha$ as a function of $p$. However, this will not produce an exact analytical expression. Instead, it is more practical to obtain $p$ as an analytical function of $\alpha$ instead, and find out the resultant implications parametrically. From the quadratic Eq. (16), $p$ is solved as two functions:

$$
p_{ \pm}=2 \sqrt{k \alpha}+\frac{w(æ-\alpha)-\frac{\eta^{2}}{2}\left(1 \pm \sqrt{\frac{4 w(\alpha-æ)}{\eta^{2}}+1}\right)}{\alpha(æ-\alpha)^{2}} .
$$

To estimate the non-dimensional parameters, we take a rather soft cell matrix that gives the mean elastic modulus $\langle\gamma\rangle \approx 1 \mathrm{kPa}$ (this value can vary from $1 \mathrm{kPa}$ to 10 $\mathrm{kPa}$ in experiments 17 (37/38), and use the bending stiffness of a MT from Gittes et al. 12: $K \approx 2 \times 10^{-23}$ $\mathrm{Nm}^{2}$ as an example. The elastic strength of a single confinement is calculated from the relation $\langle\gamma\rangle=\varepsilon / \xi$, where the mesh size $\xi$ of the intracellular matrix was found to be between 30 and $60 \mathrm{~nm}^{39 / 40}$. Yet, we deliberately let the mesh size $\xi$ be a varying parameter, not restricted to the experimentally-observed range. We then have an adjustable $\varepsilon$ and therefore an adjustable disorder strength $\eta=\Re \varepsilon / 2$, to examine the limiting case of the Winkler model. Under the room temperature $300 \mathrm{~K}$ and
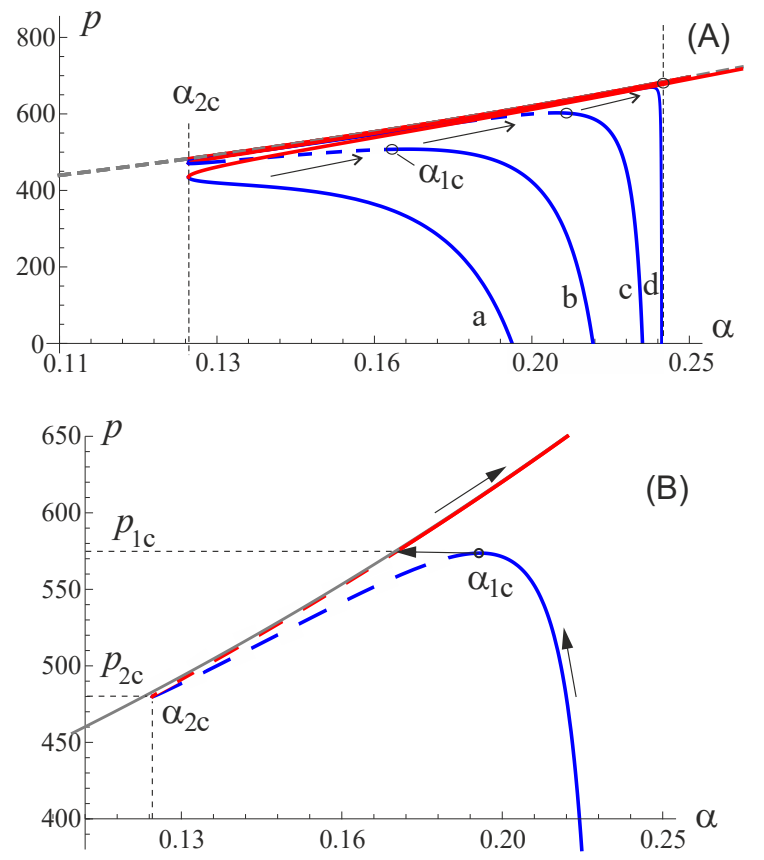

FIG. 4. (A) $p_{ \pm}(\alpha)$ at varied disorder strength $\eta$ with $k=$ $4.8 \times 10^{5}$ and $æ=0.24: p_{+}$and $p_{-}$are plotted in blue and red, respectively. The thick grey dashed line is the asymptote for $p_{-}, p=2 \sqrt{k \alpha}$. Its intersection with the vertical asymptote of $p_{+}$, i.e. $\alpha=æ$, gives the Winkler result $p=2 \sqrt{k æ}$. The curves from (a) to (d) are plotted with $\eta=5.8,2.9,1.5$ and 0.03 (corresponding to the mesh size $\xi=200,100,50$ and $1 \mathrm{~nm}$ ). The peak of $p_{+}$is shown as a circle, which disappears at strong disorder, see curve (a), but when present, it gradually reduces to the Winkler result as the disorder strength declines, indicated by the arrow. $\alpha_{1 c}$ are $\alpha_{2 c}$ are buckling points at first- and second-order transitions. (B) The zoom-in picture at the peak of the curve (c) to show the discrete jump of $\alpha$ at the first-order buckling transition. The arrow indicates how the $\alpha(p)$ solution moves with increased compression. $p_{1 c}$ and $p_{2 c}$ are critical buckling forces of the first and second-order transitions, respectively.

the length scale $\zeta=10 \mathrm{~nm}$ (chosen to roughly be the size of the tubulin dimers along the MT axis ${ }^{34}$ ), these parameters give the non-dimensional expressions $k \approx 4.8 \times 10^{5}$ and $æ \approx 0.24$, see Eq. (7). Note that $w$ is not independent of $\eta: w=\eta^{2} / 2 æ$. With these non-dimensional parameters, we plot the solution $p(\alpha)$ in Fig. 4

The $p_{+}$function originally has two branches, one of which stays in the regime $2 \sqrt{k \alpha}-p<0$, making $B$ integral in Eq. (18) physically meaningless, and therefore has been discarded (not shown in Fig. 4). The $p_{-}$function has the asymptote of $p=2 \sqrt{k \alpha}$ (by observing no divergence at $æ=\alpha$ and then taking the limit $\alpha \rightarrow \infty$ ), and governs the high- $p$ regime. If the $\alpha$ solution along this $p_{-}$function is put back into the correlator $G_{a b}^{-1}$ of Eq. 12 (with $\lambda$ there replaced with $(\alpha-æ) / 2$ and letting $\sigma=\sigma_{0}=0$ ), it almost gives a zero value, indicating that filament deflection can be very large. Thus, $p_{-}$is recognized to be the post-buckling regime. 
To investigate the behavior before/at buckling, the $p_{+}$ function in Eq. (19) is the focus. At $p=0$, the effective elastic modulus $\alpha$ is smaller than the average modulus $æ$, as $p_{+}$function always sits to the left of its vertical asymptote $\alpha=æ$. An increase in $p$ along the $p_{+}$function gradually decreases $\alpha$. Since $p_{-}$has been recognized as the post-buckling regime, the initiation of filament buckling is thought of as the switch of $\alpha$ solution from $p_{+}$to $p_{-}$that would exhibit different mechanical behavior.

To find out this critical compression for buckling, we observe how $p_{+}$evolves to give the Winkler result in Fig. 4A. We remind that the Winkler model is the case where the disorder strength $\eta$ goes to zero. It requires the mesh size $l_{m}$ and the elastic strength of one confinement $\Delta$ to be extremely small, while holding their ratio a constant, so that mean elastic modulus æ will not vanish in the relation $æ=\Delta / l_{m}$ (cf. Eq. (7) for definitions of non-dimensional parameters). The Winkler result gives the critical buckling force $p_{c}=2 \sqrt{k æ}$, see the discussion of $\vartheta$ below Eq. (12).

We notice that the peak of $p_{+}$is the only unique point that moves towards the Winkler result as disorder strength decreases. Consequently, it is recognized as the critical buckling point, giving a sudden jump from the $p_{+}$branch to the post-buckling regime $p_{-}$. The overall $\alpha$ solution path is shown as a solid line in Fig. $4 \mathrm{~B}: \alpha$ gradually decreases as $p$ increases and has a sudden jump at the buckling transition $\alpha_{1 c}$. This discrete jump of $\alpha$ is analogous to the first-order phase transition, and was also seen in the buckling of semi-flexible filaments under thermal fluctuations $\frac{11}{11}$, although the model there did not include the effect of the embedding matrix.

We also notice that the peak of $p_{+}$disappears at stronger disorder, see the curve (a) in Fig. 4. In this scenario, there is only one single-valued $\alpha$ solution as $p$ increases. The $\alpha$ solution path will no longer show a discontinuous jump. The switch from $p_{+}$to $p_{-}$becomes continuous, while maintaining the feature of a gradual decreased $\alpha$ upon increased compression, before this switch occurs. An analogy between the disappearance of the $\alpha$ jump and the critical point in the phase transitions could be drawn. Here, the sudden jump in $\alpha$ (at weak disorder) resembles the first-order phase transition. At strong disorder, although $\alpha$ is continuous, the derivative $d \alpha / d p$ is discontinuous at the transition point of $p_{+}$to $p_{-}$, hence the filament buckling in this regime is recognized as the second-order phase transition.

\subsection{Critical value of the effective elastic modulus}

In the weak disorder case, the approximation for the critical value, $\alpha_{1 c}$, can be obtained by Taylor-expanding $p_{+}$of Eq. (19) around $\alpha=æ$ to the third order, which then is used to solve the equation $d p_{+} / d \alpha=0$ and find out the peak position of $p_{+}$. For strong disorder, the switch happens at the connection point of these two $p$ functions of Eq. (19), described by the equation $p_{+}=p_{-}$ that is analytically solvable to give the exact expression of $\alpha_{2 c}$. The expressions for $\alpha_{1 c}$ and $\alpha_{2 c}$ take the form:

$$
\begin{aligned}
\frac{\alpha_{1 c}}{æ} \approx 1-\left(\frac{2 \eta^{2}}{æ^{7 / 2} k^{1 / 2}}\right)^{1 / 3} & =1-\left(\frac{\Delta^{2}}{2 æ^{3 / 2} k^{1 / 2}}\right)^{1 / 3} ; \\
\frac{\alpha_{2 c}}{æ} & =\frac{1}{2},
\end{aligned}
$$

where the subscript 1 and 2 refer to the points of first- and second-order transition, respectively. These two critical expressions of $\alpha$ can be inserted back into Eq. (19), to find out the critical buckling force (explored in the next section).

When transforming Eq. 201 back into the dimensional form, the factor of the inverse thermal energy $\beta$ cancels off on both sides. The decrease of $\alpha_{c}$ is purely due to the quenched disorder (from the disorder strength $\eta$ ), rather than the thermal effect. This result is intuitive and reasonable, as the structure of the matrix is quenched and will not re-distribute itself by thermal fluctuations, therefore exhibiting no thermal effects in the effective elasticity of the matrix. Since $\alpha_{c}$ does not depend on temperature, it also follows that the trigger of the critical phenomenon (the cross-over from $\alpha_{1 c}$ to $\alpha_{2 c}$ ) is not due to the thermal effects and is induced purely by the quenched disorder.

We plot Eq. 20 in Fig. 5 with three $æ$ values that sit within the experimental range from 1 to $10 \mathrm{kPa}$. For clear comparison, we choose the mesh size $l_{m}=\Delta / æ$ as the variable, and the result is also expressed in scaled $\alpha_{c} / æ$. A larger mesh size with a constant $æ$ indicates a higher disorder strength (from the relation $\eta=æ^{2} l_{m} / 2$ ). At $l_{m}=0$ (no disorder), Eq. 20) is simply reduced to the Winkler model, $\alpha_{1 c}=æ$. An increase in the disorder strength gradually decreases the $\alpha_{1 c}$ value, as the disorder has already been known to cause the softening of the elastic matrix in the $\alpha$ solution path in Section 3.2 . It is also noticeable that the matrix with a higher mean elastic modulus æ is more sensitive to the disorder effect of expanding the mesh size, due to the factor of $æ^{2}$ in disorder strength. Physically speaking, at a fixed mean mesh size, a higher mean elastic modulus indicates a stronger elastic support from the confinement. Any change of the spacing between two confinements due to the random distribution can have a more dramatic effect in the local elastic modulus, hence stronger disorder.

We note that the decrease in $\alpha_{c}$ from increasing disorder strength is not limitless. Beyond the threshold $\Delta_{c}$, $\alpha_{c}$ switches form $\alpha_{1 c}$ to $\alpha_{2 c}$ and stays as a constant, no matter how disordered the matrix further becomes, see the dashed horizontal line in Fig. 5 . The threshold $\Delta_{c}$ for the cross-over from $\alpha_{1 c}$ to $\alpha_{2 c}$ can be roughly estimated by equating $\alpha_{1 c}=\alpha_{2 c}$, which gives:

$$
\Delta_{c} \approx \frac{1}{2} \sqrt[4]{k æ^{3}}=æ \cdot \frac{1}{2} \sqrt[4]{\frac{k}{æ}},
$$

where the expression, $0.5(k / x)^{1 / 4}$, has the physical meaning of the mesh size, and is similar in structure to the buckling wavelength given in the Winkler model, 


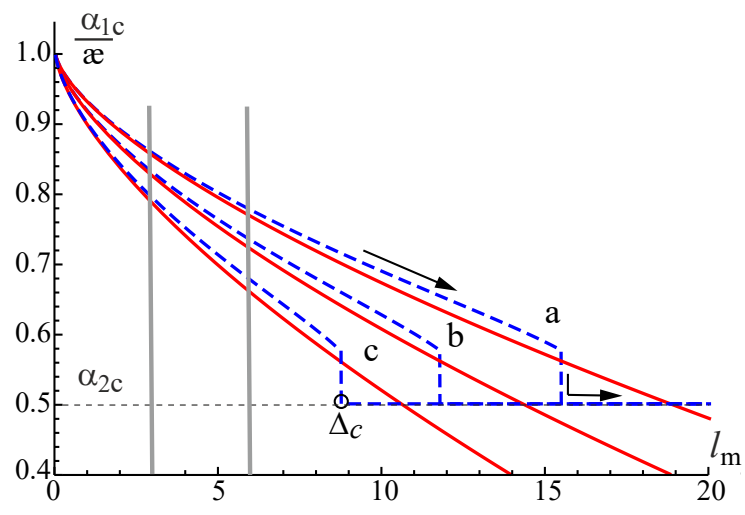

FIG. 5. The normalized $\alpha_{c}$ plotted against the nondimensional mesh size $l_{m}$, with $k=4.83 \cdot 10^{5}$ and $æ=0.24$ (set a, $1 \mathrm{kPa}), 0.72(\mathrm{~b}, 3 \mathrm{kPa})$ and $2.4(\mathrm{c}, 10 \mathrm{kPa})$. In particular, the range of the mesh size observed in the intracellular matrix is indicated by two grey lines, from $l_{m}=3$ to 6 . Each set $(\mathrm{a}, \mathrm{b}, \mathrm{c})$ contains three curves. The dashed curve is the numerical solution of $\alpha_{c}$, with the critical point is indicated by $\Delta_{c}$. The solid line is based on the approximated $\alpha_{1 c}$ given in Eq. 20, while $\alpha_{2 c}$ is shown in the dashed horizontal line, and is universal for these three sets, since $\alpha_{2 c}$ is normalized by æ. As an example, the arrows in the set (a) are used to show the flow of $\alpha_{c}$ with increasing mesh size.

$2 \pi(k / \alpha)^{1 / 4}$, yet shorter. The approximation for $\Delta_{c}$ is rather crude, but it has the benefit to give 'the upper boundary' for $\Delta_{c}$, as shown in Fig. 5 . Qualitatively speaking, the approximate Eq. (21) shows that a less rigid filament or a stronger elastic matrix is easier to induce the critical phenomenon by increasing the mesh size from the homogeneous limiting case $\left(l_{m}=0\right)$.

As one may be concerned that the critical phenomenon may originate from the failure of our continuum model for the discretized Hamiltonian, this expression enables the self-check of our assumption of slow-varying deflections at the critical phenomenon, by comparing the most dominant buckling wavelength with the mesh size. The details are moved to Appendix $\mathrm{D}$, and we find that the critical phenomenon we discover satisfies this requirement and our continuum model used for the discretized Hamiltonian still holds validity.

\subsection{Critical buckling force}

The critical buckling force in the weak and strong disorder regimes (associated with the discontinuous, and the continuous buckling transition, respectively) can be calculated by inserting $\alpha_{c}$ of Eq. 20p into $p_{+}(\alpha)$ of Eq. (19):

$$
\begin{aligned}
& \frac{p_{1 c}}{2 \sqrt{k æ}} \approx 1-\left(\frac{\eta^{2}}{32 k^{1 / 2} æ^{7 / 2}}\right)^{1 / 3}=1-\frac{\Delta^{2 / 3}}{2^{7 / 3} k^{1 / 6} æ^{1 / 2}} ; \\
& \frac{p_{2 c}}{2 \sqrt{k æ}}=\frac{1}{\sqrt{2}}-\frac{\eta^{2}}{k^{1 / 2} æ^{7 / 2}}=\frac{1}{\sqrt{2}}-\frac{\Delta^{2}}{4 k^{1 / 2} æ^{3 / 2}},
\end{aligned}
$$

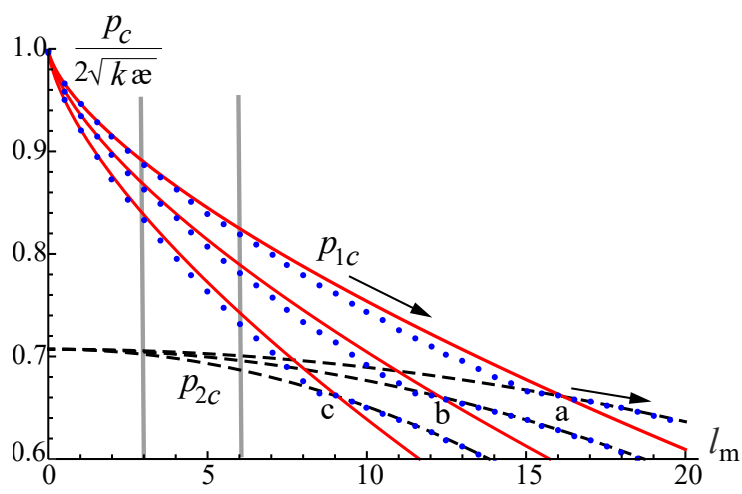

FIG. 6. Normalized plot of $p_{c}$ against the mesh size $l_{m}$, with $k=4.83 \cdot 10^{5}$ and $æ=0.24$ (set a, $\left.1 \mathrm{kPa}\right), 0.72(\mathrm{~b}, 3 \mathrm{kPa})$ and $2.4(\mathrm{c}, 10 \mathrm{kPa})$. The range of the mesh size in the intracellular matrix is the regime between two grey vertical lines. Each set $(\mathrm{a}, \mathrm{b}, \mathrm{c})$ has three curves. The numerical solutions are presented as dotted lines. The approximated expression $p_{1 c}$ given in Eq. 22 is solid lines, while $p_{2 c}$ curves are presented as dashed lines. The critical point occurs roughly around the intersection of the $p_{1 c}$ and $p_{2 c}$ lines. The arrows in the set (a) are used to show this cross-over of $p_{c}$, as an example.

where $p_{1 c}$ is the approximated expression obtained from the Taylor expansion in terms of the disorder strength $\eta$. Figure 4 illustrates the positions of $p_{1 c}$ and $p_{2 c}$ in the $\alpha$ solution path. These two $p_{c}$ relations cross over from one to the other at a point $\Delta_{c}$ in Eq. (21), when plotted against the mesh size $l_{m}$ for each specified elastic modulus æ, as shown in Fig. 6. using the same parameters and variables as Fig. 5. A somewhat better estimation of the crossover threshold, which corresponds to the critical point in the phase-transition language, can be obtained by solving the equation $p_{1 c}=p_{2 c}$. However, it gives a cubic equation for $\Delta_{c}$, and it is not worth examining, given all our approximation.

Both $p_{1 c}$ and $p_{2 c}$ decrease when the disorder strength $\eta$ increases (i.e. the mesh size $l_{m}$ expands, or the confinement strength $\Delta=l_{m} æ$ reinforces). For $p_{1 c}$, this disorder effect adds a correction term to the Winkler result $2 \sqrt{k æ}$. This is due to the softening of the matrix (the effective modulus $\alpha$ is declining with increased compression), with $\alpha_{1 c}$ put back in Eq. (19). The first term there is a reminder of the Winkler result, but the average elastic modulus $æ$ is replaced with the 'effective' modulus $\alpha$, while the second term is a complicated combination of $\alpha$ and also the disorder strength $\eta$ itself (recall that $\left.w=\eta^{2} / 2 \Delta\right)$. Both of these contributions are important in the first-order buckling. When divided by the Winkler result, the ratio of the disorder term has the scaling of $\Delta^{2 / 3}$, with the bending rigidity $k$ in the denominator, working to counter the effect of disorder. Similar to the discussion on the disorder effect in Fig. 5 of $\alpha_{1 c}$, a higher elastic modulus with a constant mesh size gives a stronger disorder, measured by the factor $\mathfrak{x}^{2} l_{m}$. Hence, decrease in the critical buckling force $p_{1 c}$ is more pronounced for a matrix with stronger elasticity, as we see Fig. 6 . 
As for the continuous buckling transition at $p_{2 c}$, although $\alpha_{2 c}$ is a constant value of $æ / 2$ after the critical point, the second term of Eq. 19 can still account for the disorder effect when increasing the disorder strength, while the first term remains unchanged. Overall, a similar result is recovered, with a different numerical factor in front of the Winkler expression and a larger scaling exponent of $\Delta$ in the disorder term: $\Delta^{2}$. The bending rigidity and the mean elastic modulus also play the same role as in the $p_{1 c}$ case, yet with larger scaling exponents. It indicates that the change in the buckling force in the second-order transition regime is more sensitive to both intrinsic $(k)$ and extrinsic $(æ$ and $\Delta)$ mechanical parameters.

At very large disorder strength, measured by the magnitude of $\eta=æ \Delta / 2$, the expression for $p_{2 c}$ loses validity, so the possible zero or even negative critical buckling force are certainly not physical. The replica-symmetrical solution that we are using here does not remain stable at very high disorder strength $28 / 41$. We are not interested in this regime because it is well outside the range of reasonable parameters in cytoskeleton network: the mesh size is between 30 to $60 \mathrm{~nm}{ }^{39 \mid 40}$ and the elastic modulus is of a few $\mathrm{kPa}^{17 / 37 / 38}$. The three red curves confined by grey lines in Figs. 5 and 6 cover this in-vivo range, all falling in the first-order transition regime and away from strong disorder limit.

Consequently, buckling of MTs in vivo resembles the first-order transition, with $p_{c}$ decreased to the fraction between 0.9 and 0.75 of the Winkler prediction. The critical buckling wavelength is estimated by the relation $\lambda_{c}=2 \pi\left(2 k / p_{c}\right)^{1 / 2}$, derived in Appendix D. Eq. (D2). Since $p_{c}$ in our model is lower than the Winkler result, the buckling wavelength $\lambda_{c}$ is accordingly longer.

Equation 22 can be transformed back into the dimensional form, giving:

$$
\begin{aligned}
& \frac{P_{1 c}}{2 \sqrt{K\langle\gamma\rangle}} \approx 1-\frac{\varepsilon^{2 / 3}}{2^{7 / 3} K^{1 / 6}\langle\gamma\rangle^{1 / 2}} ; \\
& \frac{P_{2 c}}{2 \sqrt{K\langle\gamma\rangle}}=\frac{1}{\sqrt{2}}-\frac{\varepsilon^{2}}{4 K^{1 / 2}\langle\gamma\rangle^{3 / 2}} .
\end{aligned}
$$

The expressions for the critical buckling force $P_{1 c}$ and $P_{2 c}$ do not depend on temperature. It is known that thermal fluctuations can affect the $P_{c}$ expression in the semi-flexible filament 11 . In our free energy formulation, we did include the thermal factor $\beta$ in the exponent of the partition function in Eq. (5), so one may expect our $P_{c}$ expression should also manifest the thermal effect. However, we have to take a closer look at the origin of the thermal effcts on $P_{c}$ in Ref!11. The free energy they derived actually had a different physical meaning from ours. They were able to specifically calculate the free energy under the displacement constraint ( $\Delta L=$ constant), so that they could recognize at which displacement value the most probable state occurs, and whether more than one stable state exist (and what is the barrier between them).

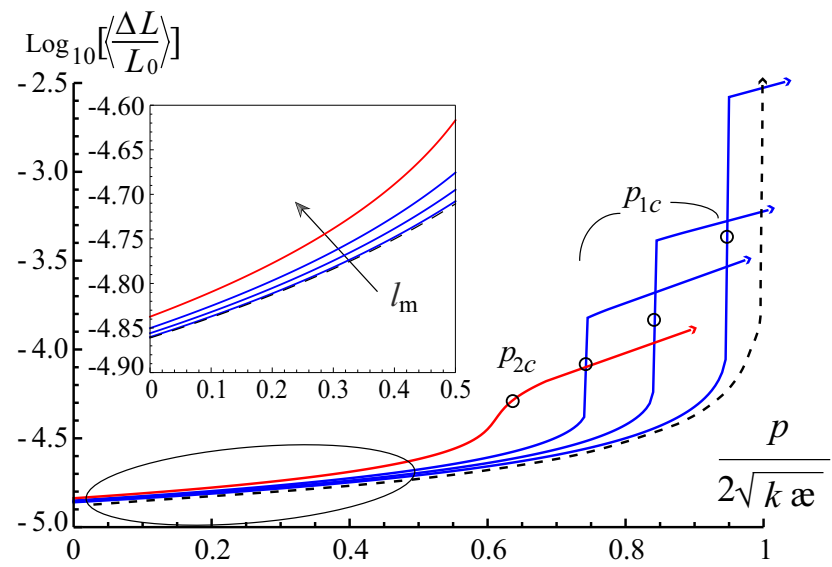

FIG. 7. Log-plot of the normalized displacement $\langle\Delta L\rangle$ against compression $p$, with $k=4.8 \cdot 10^{5}$ and $æ=0.24$, at $l_{m}=1,5,10$ (blue from right to left) and 20 (red). The Winkler result is shown in dashed line. The circle at each curve is the critical buckling point, which gradually evolves from right to left, and eventually switches from the $p_{1 c}$ regime to $p_{2 c}$, as the mesh size $l_{m}$ (the measure of disorder strength) increases. The inset is the zoom-in at small compression, where the quenched disorder already affects the displacement at zero compression.

On the other hand, the free energy we obtain here does not have this displacement constraint, and is the Gibbs free energy at constant applied compression calculated by including all the possible states of varied $\Delta L$ values. This consequently blurs the information on the free energy barrier between two or multiple possible stable states, in terms of varied $\Delta L$ values. As a result, it prevents us from further exploring how compressive force will reduce this barrier to be comparable with thermal energy and hence induce buckling. This is the limitation of our (Gibbs) formalism.

We will not state that temperature does not have any effect on $P_{c}$ in buckling inside a quenched elastic matrix. But such thermal effect is expected to be small, when the thermal energy can hardly bend single elastic confinements. In this context, our $P_{c}$ expressions in Eq. (23) show that the quenched disorder of the confinements inside an elastic matrix is a key athermal factor that affects the critical buckling force.

\subsection{Force-displacement profile}

The filament contraction $\Delta L$ under the smalldeflection assumption we used in Eq. (2) is: $\Delta L=\frac{1}{2} \int d S(d Y / d S)^{2}$, which can easily be nondimensionalized (following the same procedure in the end of Sec. 2.2, recast in the Fourier space, and re-written as a continuous integral over the wavenumber $q: \Delta L / L_{0}=l_{0} \int d q q^{2} \bar{y}_{q}^{2} / 2 \pi$. The quenched averaged displacement $\langle\Delta L\rangle$ by the definition from Eq. (5) is obtained using the average $\left\langle\bar{y}_{q}^{2}\right\rangle$, calculated in Eq. (D1). 
Appendix E gives the detail of $\langle\Delta L\rangle$ calculation:

$$
\begin{aligned}
& \left\langle\frac{\Delta L}{L_{0}}\right\rangle=\frac{1}{4 \sqrt{k}} \frac{1}{(2 \sqrt{k \alpha}-p)^{1 / 2}} \\
& \approx \frac{k_{B} T}{4 \sqrt{K}}\left(\frac{1}{\sqrt{2 \sqrt{K\langle\gamma\rangle}-P}}+\frac{\varepsilon \sqrt{K}}{4(2 \sqrt{K\langle\gamma\rangle}-P)^{2}}\right),
\end{aligned}
$$

where $\alpha(p)$ is the function discussed in Section 3.2 . Eq. (19). The second line in Eq. (24) is the approximation at weak disorder and low compression.

Figure 7 shows the force-displacement profile with a fixed value of the mean elastic modulus $æ$ and bending rigidity $k$ in each curve, while varying the disorder strength (measured by $l_{m}$ ). It has several features: the disorder strength shifts the critical buckling force from the Winkler result to a smaller value, and the discrete jump in the displacement (induced by the discontinuity in the $\alpha$ solution path, blue curve) will eventually disappear and become continuous (red curve). This is the conclusions we discussed in Sections 3.2 and 3.4 . On the other hand, the inset in Fig. 7 shows the approximate range of filament bending before the buckling threshold, where the second line of Eq. 24 can be applied. This approximation of the displacement gives two types of filament undulation that exist even at zero compression, and importantly, both of them have a thermal factor $k_{B} T$.

The first type of the undulation is purely due to thermal fluctuations, and this effect has also been seen in other filament stretching or compressing phenomenon investigated with the similar free energy calculation method 1125 . Yet, the earlier work only examined a filament without an elastic foundation, and gave this thermal term as proportional to $k_{B} T L_{0} / K$ (at zero compression and in the small-deflection regime ${ }^{11125}$. A long filament is easily bent by thermal fluctuations. In contrast, our thermal undulation term in Eq. (24) gives the ratio $k_{B} T / K^{3 / 4}\langle\gamma\rangle^{1 / 4}$ at zero compression. The thermal energy is balanced by a combination of the elastic support and the bending rigidity, while the effect of the filament length $L_{0}$ is lost. For a long filament, the elastic constraint of the matrix is a more important factor in resisting the external compression. This observation is the conclusion Winkler reached in 1866, when investigating the critical buckling force at zero temperature.

The second type of filament undulation is a combination of both thermal and quenched disorder effects. In many other quenched disorder problems (e.g. in random local filament-curvatures ${ }^{42}$ or random environmental force $e^{25}$ ), the disorder is entered as an independent term without coupling with thermal energy, and still exists at zero temperature. But in our model, the disorder in the CSK matrix vanishes at zero temperature.

This difference in disorder effect on filament bending can be understood by looking at our trial Hamiltonian $\tilde{H}_{0}=l_{0} \int d q\left(k q^{4}-p q^{2}+\alpha\right) \bar{y}_{q}^{2} / 2$, and realizing that the fully-stretched straight filament is the most stable state at $T=0$ in our model before the buckling occurs. In this scenario, the disorder embedded inside the effective elastic modulus $\alpha$ will therefore not be present. In contrast, in the cases of disordered local filament curvatures, or the random environmental force, the most stable state at $T=0$ is not the fully-stretching state, and depends on the detailed configurations of the disorder variables. It consequently gives the quenched-disorder dependence of the displacement, regardless of the presence of thermal effects.

\section{CONCLUSIONS}

We examine the buckling of a long filament embedded inside an inhomogeneous elastic matrix, where the quenched disorder originates from the distribution of confinements along the filament. We find that an analogy with first- and second-order phase transitions can be drawn in the buckling behavior, with the order parameter being the effective elastic modulus. This order parameter is found to be temperature-independent. The switch from the first-order to second-order transition, i.e. the critical point, is induced purely by the strength of quenched disorder.

In either type of buckling, the quenched disorder in elastic matrix is found to shift the athermal critical buckling force from the Winkler result $P_{c}=2 \sqrt{K\langle\gamma\rangle}$ to a lower value. Hence, the disorder inside the matrix deteriorates its ability to support and stabilize the filament. This destabilization is also seen in the filament bending at non-zero temperature before buckling. Specifically for MTs in vivo, $P_{c}$ is reduced to the fraction between 0.9 and 0.75 of the Winkler prediction, with buckling behaviour resembling the first-order phase transition. This effect, of random disorder destabilizing the effective elastic matrix, has been seen before in different context; for example, in the scaling analysis of Cates \& Ball ${ }^{22}$ it is very clear how (rare) regions of particularly weak confinement would contribute disproportionally to the effective matrix. Analytically, this effect is due to the leading quenched-disorder contribution to the replica Hamiltonian (6) before buckling is negative (as was also seen in slightly different context in ${ }^{23132}$ ).

The methodology we present in this paper is quite general. Although we assumed the Poisson distribution for matrix confinements and take the MT as an example, it should be able to extend to other distribution functions for confinements, and other ranges of parameters can also be applied, as long as we stay in the regime of a long filament. On the other hand, short filaments cannot be analyzed with the form of the correlator $G_{a b}^{-1}$ that Mézrad and Parisi have proposed for the trial Hamiltonian in the GVM. We showed that the dependence of the Fourier modes of the correlator changes, using the 4th-order Hamiltonian as an example. The buckling of a short filament in an elastic matrix needs to be investigated separately. 


\section{Acknowledgements}

This work has been supported by the Theory of Condensed Matter Critical Mass Grant from EPSRC (EP/J017639).

${ }^{1}$ D. E. Ingber, J. Cell Sci. 104, 613 (1993).

${ }^{2}$ M. F. Coughlin and D. Stamenović, J. Appl. Mech. 64, 480 (1997)

${ }^{3}$ A. M. R. Kabir, D. Inoue, T. Afrin, H. Mayama, K. Sada, and A. Kakugo, Sci. Rep. 5, $17222(2015)$

${ }^{4}$ M. Z. Jin and C. Q. Ru, Phys. Rev. E 88, 012701 (2013)

${ }^{5}$ S. Timoshenlko and J. M. Gere, Theory of elastic instability (McGraw-Hill, N. Y., 1961).

${ }^{6}$ O. Kratky and G. Porod, Recl. des Trav. Chim. des Pays-Bas 68, $1106(2010)$

'T. Odijk, Macromolecules 28, 7016 (1995)

${ }^{8}$ K. Baczynski, R. Lipowsky, and J. Kierfeld, Phys. Rev. E 76, 061914 (2007)

${ }^{9}$ M. Emanuel, H. Mohrbach, M. Saya, H. Schiessel, and I. M. Kulic, Phys. Rev. E 79, 061907 (2007).

${ }^{10}$ D. R. Nelson, Physica A 177, 220 (1991).

${ }^{11}$ J. R. Blundell and E. M. Terentjev, Soft Matter 20, 4015 (2009)

${ }^{12}$ F. Gittes, E. Meyhöfer, S. Baek, and J. Howard, Biophys. J. 70, 418 (1996)

${ }^{15}$ S. L. Gupton, W. C. Salmon, and C. M. Waterman-Storer, Curr. Biol. 12, 1891 (2002)

${ }^{14}$ C. P. Brangwynne, F. C. MacKintosh, S. Kumar, N. A. Geisse, J. Talbot, L. Mahadevan, K. K. Parker, D. E. Ingber, and D. A. Weitz, J. Cell Biol. 173, 733 (2006)

${ }^{15}$ M. Das, A. J. Levine, and F. C. MacKintosh, Europhys. Lett. 84, $18003(2008)$

${ }^{10}$ W. L. Shan, Z. Chen, C. P. Broedersz, A. A. Gumaste, W. O. Soboyejo, and C. P. Brangwynne, Soft Matter 9, 194 (2013)

${ }^{17}$ B. Fabry, G. N. Maksym, J. P. Butler, M. Glogauer, D. Navajas, and J. J. Fredberg, Phys. Rev. Lett. 87, 148102 (2001)

${ }^{18}$ B. R. Parry, I. V. Surovtsev, M. T. Cabeen, C. S. ÓHern, E. R. Dufresne, and C. Jacobs-Wagner1, J. Cell 156, 182 (2014)

${ }^{19}$ G. T. Charras, M. Coughlin, T. J. Mitchison, and L. Mahadevan, Biophys. J. 94, 1836 (2008)

${ }^{20}$ G. Salbreux, G. T. Charras, and E. Paluch, Trends Cell Biol. 22, $536(2012)$

${ }^{21}$ S. F. Edwards and M. Muthukumar, J. Chem. Phys. 89, 2435 (1988)

${ }^{22}$ M. Cates and R. C. Ball, J. Phys. France 49, 2009 (1988)

${ }^{23}$ A. Craig, E. M. Terentjev, and S. F. Edwards, Physica A 384, $150(2007)$

${ }^{24}$ P. Benetatos and E. M. Terentjev, Phys. Rev. E 82, 13 (2010)

${ }^{25}$ P. Benetatos and E. M. Terentjev, Phys. Rev. E 84, 9 (2011)

${ }^{26} \mathrm{~V}$. Dotsenko, Introduction to the replica theory of disordered (Cambridge University Press, N. Y., 2001)

${ }^{27}$ M. Mézard and G. Parisi, J. Phys. I 1, 809 (1991)

${ }^{28}$ L. Petridis and E. M. Terentjev, J. Phys. A 39, 9693 (2006)

${ }^{29}$ S. F. Edwards and P. M. Anderson, J. Phys. F. 6, 1927 (1976)

${ }^{30}$ S. V. Fridrikh and E. M. Terentjev, Phys. Rev. E 60, 1847 (1999)

${ }^{31}$ T. Castellani and A. Cavagna, J. Stat. Mech. Theory Exp. 2005, P05012 (2005)

${ }^{32}$ P. Haronska and T. A. Vilgis., J. Chem. Phys. 101, 3104 (1994)

${ }^{33}$ G. C. Wick, Phys. Rev. 80, 268 (1950)

${ }^{34}$ E. Mandelkow and E.-M. Mandelkow, Curr. Opin. Struct. Biol. 4, 171 (1994).

${ }^{35}$ H. Felgner, R. Frank, and M. Schliwa, J. Cell Sci. 109, 509 (1996).

${ }^{36}$ F. Pampaloni, G. Lattanzi, A. Jonáš, T. Surrey, E. Frey, and E.-L. Florin, Proc. Natl. Acad. Sci. USA 103, 10248 (2006)

${ }^{37}$ T. G. Kuznetsova, M. N. Starodubrseva, and N. I. Yegorenkov, Micron 38, 824 (2007)

${ }^{38}$ N. Guz, M. Dokukin, V. Karaparthi, and I. Sokolov, Biophys. J. 107, $564(2014)$
${ }^{39}$ K. Luby-Phelps, D. L. Taylor, and F. Lanni, J. Cell Biol. 102, 2015 (1986)

${ }^{40}$ T. J. Mitchison, G. T. Charras, and L. Mahadevan, Semin. Cell Dev. Biol. 19, 215 (2008)

${ }^{41}$ Y. Y. Goldschmidt, Phys. Rev. E 61, 1729 (2000)

${ }^{42}$ P. Benetatos and E. M. Terentjev, Phys. Rev. E 81, 031802 (2010)

\section{Appendix A: Replica Hamiltonian}

We discretize Eq. (4) into segments of length $\Delta_{S}$, express the continuous functions of filament deflections and elastic modulus as $Y_{a, i}$ and $\gamma_{i}$, and then insert the probability distribution function $f[\gamma]$ of Eq. (3), to calculate the replica Hamiltonian. We particularly focus on the terms containing the local elastic modulus $\gamma_{i}$, as the rest of the terms will remain the same after completing the path integral over the configurations of local elastic modulus. The $f[\gamma]$ function is further approximated with the Stirling formula, $\ln n ! \approx(n \ln n-n)$, when expressing it as an exponential function. The path integral then takes the form:

$$
\begin{gathered}
\frac{1}{\mathcal{N}} \prod_{i}^{w} \int_{0}^{\infty} d \gamma_{i} \exp \left[\gamma_{i} \nu \ln \left(\frac{\gamma_{i}}{\langle\gamma\rangle}\right)+\nu\left(\gamma_{i}-\langle\gamma\rangle\right)\right] \\
\quad \times \exp \left(\frac{-\beta}{2} \gamma_{i} \nu \varepsilon \sum_{a} Y_{a, i}^{2}\right),
\end{gathered}
$$

where $\nu$ is defined as the ratio $\left(\Delta_{S} / \varepsilon\right)$, and $\langle\gamma\rangle$ is the mean elastic modulus. Note that the exponential in the first line is the Stirling approximation for $f\left(\gamma_{i}\right)$, while the second is the local elastic energy term from $\sum_{a} H_{a}$ of Eq. (4). The direct implementation of these integrals is not plausible. We turn to the 'steepest decent' approximation with respect to $\gamma_{i}$, for the exponent of the above equation. The stationary point $\gamma_{i}^{*}$ for this exponent is found out as:

$$
\gamma_{i}^{*}=\langle\gamma\rangle e^{-\frac{\beta \varepsilon}{2} \sum_{a} Y_{a, i}^{2}}
$$

Taylor-expansion of the exponential function of Eq. A1 around $\gamma_{i}^{*}$ in Eq. A2 to the second order gives a Gaussian form in the path integral:

$$
\begin{aligned}
& \prod_{i}^{w}\left\{\exp \left[\langle\gamma\rangle \frac{\Delta_{S}}{\varepsilon}\left(-1+e^{-\frac{\beta \varepsilon}{2} \sum Y_{a, i}^{2}}\right)\right]\right. \\
\times & \left.\frac{1}{\mathcal{N}} \int d \gamma_{i} \exp \left[-\frac{\Delta_{S}\left(\gamma_{i}-\langle\gamma\rangle\right)^{2}}{2 \varepsilon\langle\gamma\rangle} e^{-\frac{\beta \varepsilon}{2} \sum Y_{a, i}^{2}}\right]\right\} .
\end{aligned}
$$

The first line of Eq. A3 now has no $\gamma_{i}$ variable, and is part of our final result of the replica Hamiltonian. But this term has another exponential inside the exponent, and therefore is still too complicated for later GVM calculations in Section 2.3. Because of our smalldeflection assumption before buckling occurs, we can Taylor-expand this exponent in terms of the summed deflections of all replicas $\sum_{a} Y_{a, i}^{2}$, and keep it to the 6 th 
order. The final result is further expressed as a continuous integral of $d S$, by assuming slowly-varying functions of deflections within each segment:

$$
\begin{gathered}
\prod_{i}^{w} \exp \left[\langle \gamma \rangle \frac { \Delta _ { S } } { \varepsilon } \left(-1+e^{\left.\left.-\frac{\beta \varepsilon}{2} \sum Y_{a, i}^{2}\right)\right]}\right.\right. \\
\approx \exp \left\{\frac { - \beta \langle \gamma \rangle } { 2 } \int d S \left[\sum Y_{a}^{2}-\frac{\varepsilon}{4}\left(\sum Y_{a}^{2}\right)^{2}\right.\right. \\
\left.\left.+\frac{\beta \varepsilon^{2}}{24}\left(\sum Y_{a}^{2}\right)^{3}\right]\right\} .
\end{gathered}
$$

We go back to the second line of Eq. A3, which contains an apparently Gaussian path integral and the normalization factor $\mathcal{N}$. This normalization factor can be calculated using the same 'steepest descent' method. The overall result takes the form:

$$
\begin{aligned}
& \frac{1}{\mathcal{N}} \prod_{i}^{w} \int_{0}^{\infty} d \gamma_{i} \exp \left[-\frac{\Delta_{S}\left(\gamma_{i}-\langle\gamma\rangle\right)^{2}}{2 \varepsilon\langle\gamma\rangle} e^{-\frac{\beta \varepsilon}{2} \sum Y_{a, i}^{2}}\right] \\
\approx & \prod_{i}^{w} \frac{\int d \gamma_{i} \exp \left[-\frac{\Delta_{S}\left(\gamma_{i}-\langle\gamma\rangle\right)^{2}}{2 \varepsilon\langle\gamma\rangle} e^{\left.-\frac{\beta \varepsilon}{2} \sum Y_{a, i}^{2}\right]}\right.}{\int d \gamma_{i} \exp \left[-\frac{\Delta_{S}\left(\gamma_{i}-\langle\gamma\rangle\right)^{2}}{2 \varepsilon\langle\gamma\rangle}\right]} \\
= & \exp \left\{\sum_{i} \ln \frac{1+\operatorname{erf}\left(\sqrt{e^{\varphi_{i}}\langle\gamma\rangle \Delta_{S} / 2 \varepsilon}\right)}{e^{\varphi_{i} / 2}\left[1+\operatorname{erf}\left(\sqrt{\left\langle\gamma \Delta_{S} / 2 \varepsilon\right.}\right)\right]}\right\},
\end{aligned}
$$

where $\varphi_{i}=\frac{1}{2} \beta \varepsilon \sum_{a} Y_{a, i}^{2}$ and erf means the error function. Because of the small-deflection assumption, we Taylorexpand the ln-term in terms of $\varphi_{i}$, and find out that each term of this series has a decaying exponential factor, $\exp \left(-\Delta_{S}\langle\gamma\rangle / 2 \varepsilon\right)$. As $\Delta_{S}$ increases, Eq. A5 will eventually reduce to one and play no role in the replica Hamiltonian. This indicates that this ln-term is the error produced when we apply the 'steepest descent' approximation and express the original integral in the Gaussian form, as it is well-known that the Poisson distribution can be replaced by the Gaussian form only when the step size (the interval) is large enough.

Because this decaying exponential factor appears in the exponent of Eq. A5, it, in fact, decays rather rapidly as $\Delta_{S}$ increases. There should be a suitable $\Delta_{S}$ value that makes this error become negligible, while the transformation into the continuum model is still applicable. In this sense, Eq. A1) is approximately equal to Eq. A4). We take out the thermal factor, $-\beta$, in the exponent of Eq. A4, and together with the original terms that do not contain the local elastic modulus $\gamma(S)$, i.e. the bending energy and work done by compression, it gives the replica Hamiltonian of Eq. (6).

\section{Appendix B: Variational free energy}

In this section, we use the 4th-order case as an example, to show the origins of the pre-factor $\Gamma_{4}$ in Eq. (8), and then how to remove this $\Gamma_{4}$ factor by paring the
Fourier mode to obtain the variational free energy in Eq. (11). We start from inserting the Fourier series: $y_{a}=\sum_{n} \bar{y}_{a n} \sin \left(n \pi s / l_{0}\right)$ into the replica Hamiltonian of Eq. (7). The integral we are dealing with is:

$\int_{0}^{l_{0}} d s \sin \left(\frac{n_{1} \pi s}{l_{0}}\right) \sin \left(\frac{n_{2} \pi s}{l_{0}}\right) \sin \left(\frac{n_{3} \pi s}{l_{0}}\right) \sin \left(\frac{n_{4} \pi s}{l_{0}}\right)$

The product of sin-functions can be decomposed into cosfunctions, by using the identities:

$$
\begin{aligned}
& \sin A \cdot \sin B=\frac{1}{2}[\cos (B-A)-\cos (B+A)] ; \\
& \cos A \cdot \cos B=\frac{1}{2}[\cos (A+B)-\cos (A-B)] .
\end{aligned}
$$

Eventually eight cos-functions with a numerical factor $1 / 8$ in front are produced. Each of them after integration gives a Kronecker delta. For example, $\int d s \cos \left[\left(n_{1}-n_{2}+\right.\right.$ $\left.\left.n_{3}-n_{4}\right) \pi s / l_{0}\right]=l_{0} \delta\left(n_{1}-n_{2}+n_{3}-n_{4}\right)$. The final result of the integrated sin-product gives $l_{0} \Gamma_{4} / 8$, where $\Gamma_{4}$ is defined as:

$$
\begin{array}{r}
\Gamma_{4}=\delta\left(n_{1}+n_{2}+n_{3}+n_{4}\right)+\delta\left(n_{1}-n_{2}+n_{3}-n_{4}\right) \\
+\delta\left(n_{1}+n_{2}-n_{3}-n_{4}\right)+\delta\left(n_{1}-n_{2}-n_{3}+n_{4}\right) \\
-\delta\left(n_{1}-n_{2}+n_{3}+n_{4}\right)-\delta\left(n_{1}-n_{2}-n_{3}-n_{4}\right) \\
-\delta\left(n_{1}+n_{2}+n_{3}-n_{4}\right)-\delta\left(n_{1}+n_{2}-n_{3}+n_{4}\right) .
\end{array}
$$

The same procedure to integrate sin-product in the 6thorder case can be applied to find out $\Gamma_{6}$ (with 32 deltas inside), but we will not show its result here.

We move on to calculate the variational free energy defined in Eq. (10), with the trial Hamiltonian shown in Eq. (9), and look at how to remove the deltas of $\Gamma_{4}$ in the 4 th-order contributions of $\left\langle\tilde{H}_{\text {rep }}\right\rangle_{0}$, that is, $\sum_{a, b} \sum_{\{n\}}-\eta l_{0} \Gamma_{4}\left\langle\bar{y}_{a n_{1}} \bar{y}_{a n_{2}} \bar{y}_{b n_{3}} \bar{y}_{b n_{4}}\right\rangle_{0} / 32$.

The Gaussian form of $\left\langle\tilde{H}_{\text {rep }}\right\rangle_{0}$ requires the Fourier modes in $\bar{y}_{a n}$-product above to be paired, in order to produce a non-zero value. It follows that some of the terms in $\Gamma_{4}$ can never meet the pairing-requirement, if one wants to have a non-zero value from the Kronecker delta. Those with the odd number of '-' signs, e.g. $\delta\left(n_{1}-n_{2}-n_{3}-n_{4}\right)$, and $\delta\left(n_{1}+n_{2}+n_{3}+n_{4}\right)$ will be killed during the discussion. Only three deltas of $\Gamma_{4}$ remain: $\delta(1-2+3-4), \delta(1+2-3-4)$, and $\delta(1-2-3+4)$, where the shorthand $n_{1} \rightarrow 1$, and so on, is used. We will take $\sum_{\{n\}} \delta(1-2-3+4)\left\langle\bar{y}_{a 1} \bar{y}_{a 2} \bar{y}_{b 3} \bar{y}_{b 4}\right\rangle_{0}$ as an example, to show a systematic way to remove the Kronecker delta when summing over the Fourier modes $n$.

We consider two situations: all with the same Fourier modes, and two pairs with different modes. For cleanness, we will not explicitly write the summation for replica indices and Fourier modes for the following content of this section. In the first scenario, we need to calculate $\left\langle\tilde{y}_{a 1} \tilde{y}_{a 1} \tilde{y}_{b 1} \tilde{y}_{b 1}\right\rangle_{0}$, which can easily be obtained with the Wick formula:

$$
\left\langle\tilde{y}_{a 1} \tilde{y}_{a 1} \tilde{y}_{b 1} \tilde{y}_{b 1}\right\rangle_{0}=G_{a a}^{(1)} G_{b b}^{(1)}+2 G_{a b}^{(1)} G_{a b}^{(1)},
$$


where the notation $\sum_{n_{1}}$ and $\sum_{a, b}$ are omitted, following the convention we mentioned above. Next, two pairs with different modes have two possibilities:

$$
\begin{aligned}
& \left\langle\tilde{y}_{a 1} \tilde{y}_{a 1} \tilde{y}_{b 2} \tilde{y}_{b 2}\right\rangle_{0} \tau=G_{a a}^{(1)} G_{b b}^{(2)} \tau ; \\
& \left\langle\tilde{y}_{a 1} \tilde{y}_{a 2} \tilde{y}_{b 1} \tilde{y}_{b 2}\right\rangle_{0} \tau=G_{a b}^{(1)} G_{a b}^{(2)} \tau,
\end{aligned}
$$

where the factor $\tau$ is to ensure these two modes are different, $\tau=1-\delta(1-2)$. Combining Eqs. (B1) and (B2) gives the result for this example. The rest of the deltas inside $\Gamma_{4}$ and the $\Gamma_{6}$ term of Eq. (8) can be dealt with in the same procedures, to find out $H_{\text {rep }}$ and then obtain the variational free energy $\tilde{F}$. Here, we only show the final result:

$$
\begin{aligned}
& \tilde{F}=-\frac{1}{2} \sum_{n} \operatorname{Tr} \ln \underline{\underline{G}}(n)+\frac{l_{0} \psi(1)}{2} G_{a a}^{(1)} \\
& -\frac{\eta l_{0}}{32}\left(4 G_{a b}^{(1)} G_{a b}^{(2)}+2 G_{a a}^{(1)} G_{b b}^{(2)}+2 G_{a b}^{(1)} G_{a b}^{(1)}+G_{a a}^{(1)} G_{b b}^{(1)}\right) \\
& +\frac{w l_{0}}{192}\left(32 G_{a b}^{(1)} G_{a c}^{(2)} G_{b c}^{(3)}+24 G_{a a}^{(1)} G_{b c}^{(2)} G_{b c}^{(3)}+4 G_{a a}^{(1)} G_{b b}^{(2)} G_{c c}^{(3)}\right. \\
& +48 G_{a b}^{(1)} G_{a c}^{(1)} G_{b c}^{(2)}+24 G_{a a}^{(1)} G_{b c}^{(1)} G_{b c}^{(2)}+6 G_{a a}^{(1)} G_{b b}^{(1)} G_{c c}^{(2)} \\
& +12 G_{a c}^{(1)} G_{a c}^{(1)} G_{b b}^{(2)}-3 G_{a b}^{(1)} G_{b b}^{(2)} G_{c c}^{(1+2)}-6 G_{a b}^{(1)} G_{a b}^{(2)} G_{c c}^{(1+2)} \\
& \left.-12 G_{a b}^{(1)} G_{c c}^{(2)} G_{a b}^{(1+2)}-24 G_{a c}^{(1)} G_{b c}^{(2)} G_{a b}^{(1+2)}\right),
\end{aligned}
$$

where the definition of $\psi(1)$ is given below Eq. 111, and the summation over replica indices and Fourier modes that show up in individual terms are omitted. For instance, the term $G_{a b}(1) G_{a b}(2)$ is summed over $a, b$, $n_{1}$ and $n_{2}$ and $G_{a a}(1) G_{b b}(1)$ is summed over $a, b$, and $n_{1}$ (note that not over $n_{2}$ ). The superscript $1+2$ means $n_{1}+n_{2}$, and it is a result from the 6th-order case that gives the requirement $n_{1}=n_{3}, n_{2}=n_{4}$ and $n_{5}=n_{6}=n_{1}+n_{2}$, when discussing the pairing to remove $\delta\left(n_{1}+n_{2}+n_{3}+n_{4}-n_{5}-n_{6}\right)$, and other similar deltas.

For a long filament, the full expression of $\tilde{F}$ of Eq. (B3) can be approximated by finding out $l_{0}$-scaling in each individual term, so as to recognize which of them are the major contributions, when taking the limit $l_{0} \rightarrow \infty$. The summation over Fourier modes $\sum_{n}$ is replaced with the integral $\int d n$, and we further use the wavenumber $q=n \pi / l_{0}$ as the new variable for integration, therein, $\int d n \rightarrow l_{0} \int d q / \pi$. And we notice that $G_{a b}(n)$, which can be readily obtained from its symmetrical inverse of Eq. (9), contains the $1 / l_{0}$ factor inside:

$$
G_{a b}(n)=\frac{1}{l_{0} \Lambda}\left[\delta_{a b}-\frac{\sigma}{\Lambda+m \sigma}\right] .
$$

With $q$-transformation of $\sum_{n}$ and $G_{a b}$ expression above, we are now ready to do the scaling analysis and find that, for example:

$$
G_{a b}^{(1)} G_{a b}^{(2)} \propto l_{0}^{2} * \frac{1}{l_{0}^{2}}=1 ; G_{a a}^{(1)} G_{b b}^{(1)} \propto l_{0} * \frac{1}{l_{0}^{2}}=\frac{1}{l_{0}},
$$

where the first $l_{0}$ factor on the right hand side is from the $q$-transformation $\left(l_{0}^{2}\right.$ from the double sum, $\sum_{n_{1}, n_{2}}$, and $l_{0}$ from one sum, $\sum_{n_{1}}$ ), while the second factor is from the product of two $G_{a b}(n)$. As $l_{0} \rightarrow \infty$, one can find out which terms are more prevailing. The similar analysis can be applied to the rest of terms of Eq. (B3). It turns out that the terms, with the number of sums over Fourier modes equal to the number of $G_{a b}$ inside the product, has $l_{0}$ factor, e.g. $w l_{0} G_{a b}^{(1)} G_{a c}^{(2)} G_{b c}^{(1)} / 6$ (the first term in the third line of Eq. (B3)), while other terms will have no $l_{0}$ factor, except for the term $-\sum_{n} \operatorname{Tr} \ln \underline{\underline{G}}(n) / 2$ which we will have to discuss separately.

If the matrix has the form: $M_{a b}=A \delta_{a b}-A B I_{a b}$, where $\delta_{a b}$ and $I_{a b}$ are elements of the identity matrix and the matrix of ones, respectively, and $A$ and $B$ are constants or functions independent of the matrix indices $a$ and $b$. $\ln \underline{\underline{M}}$ can be expressed as:

$$
(\ln \underline{\underline{M}})_{a b}=(\ln A) \delta_{a b}+\frac{1}{d} \ln (1-d B) I_{a b},
$$

where $d$ is the dimension of the matrix. The trace of $\ln \underline{\underline{M}}$ above is simply:

$$
\operatorname{Tr} \ln \underline{\underline{M}}=d \ln A+\ln (1-d B) .
$$

In fact, our correlator $\underline{\underline{G}}$ of Eq. (14) has the same structure as $\underline{\underline{M}}$ above, and we can use Eq. (B6) to calculate $\operatorname{Tr} \ln \underline{\underline{G}}$. Recall that our purpose here is simply find the $l_{0}$ scaling in $\sum_{n} \operatorname{Tr} \ln \underline{\underline{G}}(n)$. We recognize that the $A$ factor in Eq. B5 has the scaling $1 / l_{0}$ in the case of our correlator $\underline{\underline{G}}$ from Eq. (B4), while $B$ does not contain any $l_{0}$ factor. $\sum_{n} \operatorname{Tr} \ln \underline{\underline{G}}(n)$ takes the form $\sum_{n}\left(-d \ln l_{0}+C\right)$, where $C$ is a function irrelevant to $l_{0}$. Following the $q$-transformation for the $n$-sum, $\sum_{n} \operatorname{Tr} \ln \underline{\underline{G}}(n) \propto\left(d l_{0} \ln l_{0}-C l_{0}\right)$, and it is therefore one of the major contributions in Eq. (B3). Overall, the major contributions of the variation free energy in the long filament case is presented in Eq. (11) in the main content. As for the short-filament case, one has to use the full expression of the variational free energy, Eq. (B3).

\section{Appendix C: Optimization equation}

In this section, we start from the approximate variational free energy in the long filament case, Eq. (11), find out its optimization equation with respect to $G_{i j}$, and show the $n$-dependence inside $\Lambda$ of Eq. (9). Then we discuss the short-filament case to show that this $n$ dependence will be changed, from its optimization equation. We will not present the full calculation detail to obtain the optimization equation in the short-filament case, but instead equip the readers with the necessary tools used through this procedure. 
The optimization equation is obtained from the condition $\delta \tilde{F} / \delta G_{i j}(n)=0$ (using Eq. (11) for the long-filament case), and further simplified by taking the limit: the total number of replicas goes to zero, $m \rightarrow 0$. We introduce a useful identity for the calculation of this derivative $\delta \tilde{F} / \delta G_{i j}(n)$, particularly for terms of $\tilde{H}_{\text {rep }}$ inside $\tilde{F}$ :

$$
\frac{\delta G_{a b}\left(n_{1}\right)}{\delta G_{i j}(n)}=\delta_{i a} \delta_{j b} \delta_{n_{1} n},
$$

where these deltas are Kronecker deltas, and note that the summation convention for repeated indices is not used here. We calculate the terms of the second line in Eq. (11) as an example:

$$
\begin{aligned}
\sum_{a, b} \sum_{n_{1}, n_{2}} \frac{\delta G_{a b}\left(n_{1}\right) \delta G_{a b}\left(n_{1}\right)}{\delta G_{i j}(n)} & =2 \sum_{n_{1}} G_{i j}\left(n_{1}\right) ; \\
\sum_{a, b} \sum_{n_{1}, n_{2}} \frac{\delta G_{a a}\left(n_{1}\right) \delta G_{b b}\left(n_{2}\right)}{\delta G_{i j}(n)} & =2 \delta_{i j} \sum_{a} \sum_{n_{1}} G_{a a}\left(n_{1}\right) \\
= & 2 \delta_{i j} m \sum_{n_{1}} G_{11}\left(n_{1}\right),
\end{aligned}
$$

where in the last line, the replica symmetry of $G_{a b}$ can extract an $m$ factor out, and this term will vanish as taking the limit $m \rightarrow 0$. One can repeat the same procedure, keep the terms that survive after taking $m \rightarrow 0$, and eventually will reach the result:

$$
\begin{aligned}
G_{a b}^{-1}(n) & \approx l_{0} \psi(n) \delta_{a b} \\
& +\frac{l_{0}}{2}\left(w \sum_{i}^{m} \sum_{\{n\}} G_{a i}^{(1)} G_{i b}^{(2)}-\eta \sum_{n_{1}} G_{a b}^{(1)}\right) I_{a b},
\end{aligned}
$$

where $\psi(n)$ is defined below Eq. (11). We further remove the embedded $m$-dependence of Eq. (13), by inserting the replica symmetrical form of $G_{a b}$ in Eq. (B4):

$$
\begin{aligned}
\sum_{i}^{m} \sum_{\{n\}} G_{a i}^{(1)} G_{i b}^{(2)}=\sum_{\{n\}} & {\left[A(1) A(2) \delta_{a b}-2 A(1) B(2) I_{a b}\right.} \\
& \left.+m B(1) B(2) I_{a b}\right],
\end{aligned}
$$

where the shorthand $A(n)$ and $B(n)$ are the matrix elements defined by $G_{a b}(n)=A \delta_{a b}-B I_{a b}$, with the detailed $G_{a b}(n)$ expression given in Eq. (B). The $m$ factor of Eq. C3 is from the sum: $\sum_{i}^{m} I_{a i} I_{i b}$, and hence the last term will vanish at $m=0$.

We now express the optimization equation $\sqrt{\mathrm{C} 2}$ in terms of $\Lambda$ and $\sigma$, by inserting $G_{a b}^{-1}$ of Eq. (9), $G_{a b}$ of Eq. (B4) and removing $m$-dependence as shown in Eq. (C3). The diagonal element generates the optimization equation of the form:

$$
\Lambda(n) \approx \psi(n)-\frac{\eta}{2 l_{0}} \sum_{n} \frac{1}{\Lambda(n)}+\frac{w}{2 l_{0}^{2}}\left(\sum_{n} \frac{1}{\Lambda(n)}\right)^{2} .
$$

We observe that the $n$-dependence of $\Lambda(n)$ is given by $\psi(n)$, while the last two terms on the right hand side of
Eq. (C4) are simply a constant independent of $n$. We can define this constant as $\lambda$, giving $\Lambda=\psi+\lambda$, which can be put back into Eq. (9) and recover $G_{a b}^{-1}(n)$ of Eq. (12) used for a long filament (beware that $\psi$ has the same expression as $\vartheta$ ).

We now consider the short-filament case that requires the full expression of the variational free energy of Eq. (B3). We follow similar calculation procedures of the long-filament case, by using Eqs. (C1) and (B4), and following the same analysis of $m$-dependence. The final optimization equation for the diagonal part in the shortfilament case has the form:

$$
\begin{gathered}
\left\{\frac{l_{0}}{2} \psi(n)-\frac{l_{0}}{2} \Lambda(n)-\frac{\eta}{4 \Lambda(n)}+\frac{w}{2 l_{0} \Lambda(n)} \sum_{n_{1}} \frac{1}{\Lambda\left(n_{1}\right)}\right. \\
\left.-\frac{w}{4 l_{0}} \sum_{n_{1}} \frac{1}{\Lambda\left(n_{1}\right) \Lambda\left(n_{1}+n\right)}+\frac{u_{n} w}{8 l_{0}} \sum_{n_{1}=1}^{n-1} \frac{1}{\Lambda\left(n_{1}\right) \Lambda\left(n-n_{1}\right)}\right\} \\
+\left\{\frac{-\eta}{4} \sum_{n_{1}} \frac{1}{\Lambda\left(n_{1}\right)}+\frac{w}{4 l_{0}}\left(\sum_{n_{1}} \frac{1}{\Lambda\left(n_{1}\right)}\right)^{2}\right. \\
\left.+\frac{w}{4 l_{0}} \sum_{n_{1}} \frac{1}{\Lambda^{2}\left(n_{1}\right)}\right\}=0,
\end{gathered}
$$

where $u_{n}$ is a step function that equals to one when $n \geq 2$, otherwise zero, and is a result when calculating $\sum_{n_{1}, n_{2}} \delta G_{a b}\left(n_{1}+n_{2}\right) / \delta G_{i j}(n)$. There, we use the change of variable $n_{3} \equiv n_{1}+n_{2}$, and the sum $\sum_{n_{1}, n_{2}=1}^{\infty}$ is transformed to $\sum_{n_{3}=2}^{\infty} \sum_{n_{1}=1}^{n_{3}-1}$ (note the change of the upper and lower limits of the sums).

Equation (C5) can recover the result of Eq. C4, following the same analysis of $l_{0}$ scaling shown in Appendix $\mathrm{B}$ to obtain the approximate variational free energy. The first bracket of Eq. C5 contains functions of $n$ and is used to investigate the $n$-dependence in $\Lambda$, while the second bracket is simply an $n$-independent terms and can be denoted as a constant $2 l_{0} \lambda$, without losing any generality.

Nevertheless, the $n$-dependence of $\Lambda$ is hard to obtain in Eq. (C5) for the 6th-order case (with the $w$ coefficient). We consider a simpler 4th-order case, letting $w=0$ in Eq. (C5), and write it in the form:

$$
\frac{l_{0}}{2} \psi(n)-\frac{l_{0}}{2} \Lambda(n)-\frac{\eta}{4 \Lambda(n)}+2 l_{0} \lambda=0,
$$

where $2 l_{0} \lambda$ is defined as a constant given by the second bracket of Eq. C5 with $w=0$ there. This equation is quadratic and we can obtain the $n$-dependence of $\Lambda$ as:

$$
\Lambda(n)=\frac{1}{2}\left[\psi(n)+\lambda+\sqrt{(\psi(n)+\lambda)^{2}-\eta / 2 l_{0}}\right] .
$$

Note that if letting $l_{0} \rightarrow \infty$, the above $\Lambda$ expression reduces to Eq. 12 for a long filament. It can also be seen that the $\Lambda(n)$ in the 4th-order case for the short filament has totally a different form and $n$-dependence. Therefore, 
for the short-filament case, our assumed $n$-dependence of $G_{a b}^{-1}$ in the main context is not applicable, and can only be used for the long filament. The following analysis and results of buckling we obtain there cannot extend to the short filaments.

\section{Appendix D: Self-check of the mesh size at critical points}

In the continuum model, a careful check should be made by comparing the non-dimensional mesh size, i.e. the spacing between confinements, with the most dominant buckling wavelength. Only when the mesh size is smaller than this buckling wavelength, the use of the continuum model is valid, as will not violate the assumption of smooth and slowly-varying deflections within the segment $\Delta_{S}$.

The non-dimensional mesh size $l_{m}$ is given as: $l_{m}=$ $\Delta / æ$, where we remind that $æ$ and $\Delta$ are non-dimensional mean elastic modulus and elastic strength of single confinement, respectively. To find out the non-dimensional wavelength $\lambda_{d}$ of the most dominant buckling mode, we need to obtain the mean-squared Fourier amplitude of mode $n_{1}:\left\langle\bar{y}_{n_{1}} \bar{y}_{n_{1}}\right\rangle$. From the quench-averaging definition given in Eq. (5) and the trial Hamiltonian of Eq. (9) with the correlator $G_{a b}^{-1}$ given in Eq. $\left[12,\left\langle\bar{y}_{n} \bar{y}_{n}\right\rangle\right.$ is calculated through:

$$
\begin{aligned}
\left\langle\bar{y}_{n_{1}} \bar{y}_{n_{1}}\right\rangle & =\lim _{m \rightarrow 0} \int\left(\prod_{a=1}^{m} \prod_{n} d \bar{y}_{a n}\right) \bar{y}_{1 n} \bar{y}_{1 n} e^{-\tilde{H}_{0}} \\
= & \lim _{m \rightarrow 0}\left\{G_{11}\left(n_{1}\right) \exp \left[\frac{1}{2} \operatorname{Tr} \ln (2 \pi \underline{\underline{G}})\right]\right\} \\
= & \frac{1}{l_{0}\left(\vartheta\left(n_{1}\right)+\lambda_{0}\right)}=\frac{2}{l_{0}\left(k q^{4}-p q^{2}+\alpha\right)},
\end{aligned}
$$

where $\operatorname{Tr} \ln (2 \pi \underline{\underline{G}})$ reduces to one, as can be checked with help of Eq. (B6) (letting $d=m=0$ and notice that $A$ and $B$ are simply a constant at $m=0$ in our $G_{a b}$ case). The definition of $\vartheta$ can be found in Eq. (12) and $\lambda_{0}$ is the solution of the optimization equation with the limit $m \rightarrow 0$, see Eq. (13). In the final expression, we use the wavenumber $q$ (defined as $n \pi / l_{0}$ ) and $\alpha$ to replace $n$ and $\lambda$.

From Eq. (D1), the most dominant wavenumber $q_{d}$, together with the corresponding buckling wavelength $\lambda_{d}$, can be obtained as:

$$
q_{d}=\sqrt{\frac{p}{2 k}} ; \quad \lambda_{d}=\frac{2 \pi}{q_{d}}=2 \pi \sqrt{\frac{2 k}{p}} .
$$

The self-check of the validity of the continuum model is to compare $\lambda_{d}$ above with the non-dimensional mesh size $l_{m}=\Delta / æ$.

We are particularly interested in investigating whether the validity of the continuum model still holds at the occurrence of the critical phenomenon. We can obtain the buckling force $\left\langle p_{c}\right\rangle$ at this critical point, by inserting
$\Delta_{c}$ of Eq. 21) into the $p_{2 c}$ expression of Eq. 222, and we subsequently put the obtained result into Eq. (D2) to find the dominant wavelength $\lambda_{d}^{c}$. It is :

$$
\lambda_{d}^{c}=4 \sqrt{2} \pi \sqrt{\frac{k}{8 \sqrt{æ k}-k^{1 / 3}\left(2 æ l_{m}^{c}\right)^{2 / 3}}},
$$

where $l_{m}^{c}$ is 'upper boundary' of the non-dimensional mesh size at the critical phenomenon and is given as $l_{m}^{c}=0.5 *(k / æ)^{1 / 4}$. The inequality to be proven is:

$$
\begin{aligned}
& \lambda_{d}^{c}=4 \sqrt{2} \pi \sqrt{\frac{k}{8 \sqrt{æ k}-k^{1 / 3}\left(2 æ l_{m}^{c}\right)^{2 / 3}}}>l_{m}^{c} \\
& \rightarrow \frac{k}{8 \sqrt{æ k}-k^{1 / 3}\left(2 æ l_{m}^{c}\right)^{2 / 3}}>\frac{\left(l_{m}^{c}\right)^{2}}{32 \pi^{2}} \\
& \rightarrow \frac{1}{7} \sqrt{\frac{k}{æ}}>\frac{1}{128 \pi^{2}} \sqrt{\frac{k}{æ}},
\end{aligned}
$$

where the last line we expand $l_{m}^{c}$ in $k$ and $æ$. This inequality always holds, so the validity of our continuum model can extend to the critical point we observe in Section 3.2 .

\section{Appendix E: Force-displacement profile}

The displacement $\Delta L$ under the small-deflection assumption we use for the filament Hamiltonian takes the form:

$$
\Delta L=\frac{1}{2} \int d S\left(\frac{d Y}{d S}\right)^{2} .
$$

The above relation can be re-written in the nondimensional form, following the same procedure in the end of Section 2.1, and then using the Fourier series $y=\sum_{n} \bar{y}_{n} \sin \left(n \pi / l_{0}\right)$, which gives:

$$
\Delta L=\frac{\zeta}{2} \int d s\left(\frac{d y}{d s}\right)^{2}=\frac{\zeta l_{0}}{4} \sum_{n} \bar{y}_{n}^{2}\left(\frac{n \pi}{l_{0}}\right)^{2},
$$

where $\zeta$ is the length scale we choose for nondimensionalization, with all other non-dimensional variables or parameters defined below Eq. (7).

The quench-averaged displacement $\langle\Delta L\rangle$ is easily obtained by combining the above equation with the relation of $\left\langle\bar{y}_{n} \bar{y}_{n}\right\rangle$ given in Eq. (D1). For a long-filament, the $n$-sum can be replaced with the $n$-integral, and then rewritten in the $q$-form (with $q=n \pi / l_{0}$ ). We obtain the relation:

$$
\left\langle\frac{\Delta L}{L_{0}}\right\rangle=\frac{1}{2 \pi} \int_{0}^{\infty} \frac{q^{2}}{k q^{4}-p q^{2}+\alpha} d q .
$$

Note that $L_{0}$ is the filament length with the relation $L_{0}=$ $l_{0} \zeta$, and the integral limits of $q$ are zero and $\infty$, due to our 
assumption of a continuous long filament. Equation E2 after integration gives:

$$
\begin{aligned}
\left\langle\frac{\Delta L}{L_{0}}\right\rangle & =\left.\frac{-A_{1} \tan ^{-1}\left(\frac{\sqrt{2 k}}{A_{1}} q\right)+A_{2} \tan ^{-1}\left(\frac{\sqrt{2 k}}{A_{2}} q\right)}{\pi \sqrt{8 k\left(p^{2}-4 k \alpha\right)}}\right|_{q=0} ^{\infty} \\
& =\frac{-A_{1}+A_{2}}{4 \sqrt{2 k\left(p^{2}-4 k \alpha\right)}}
\end{aligned}
$$

with

$A_{1}=\sqrt{-p-\sqrt{p^{2}-4 k \alpha}}$ and $A_{2}=\sqrt{-p+\sqrt{p^{2}-4 k \alpha}}$.

To simplify $-A_{1}+A_{2}$ in the numerator, we need to know whether $p^{2}-4 k \alpha$ is positive or negative, so that we can find the expressions of $A_{1}$ and $A_{2}$ in the complex plane. By looking back at Eq. 19p of $p_{ \pm}$, we can see that both of these $p$ functions sit below the asymptote $p=2 \sqrt{k \alpha}$, and therefore $p^{2}-4 k \alpha$ is always negative. This is a result coming from the restriction that the correlator $\underline{\underline{G}}^{-1}$ of our trial Gaussian Hamiltonian in Eq. 12 must be positive for all $n$ values, or the Gaussian integral becomes divergent and ill-defined. With the condition $p^{2}-4 k \alpha<0$, we find that $-A_{1}+A_{2}$ is purely a imaginary number:

$$
-A_{1}+A_{2}=\sqrt{2} \sqrt{p+2 \sqrt{k \alpha}} i \text {. }
$$

Inserting the above relation back into Eq. E3 and be careful that $\sqrt{p^{2}-4 k \alpha}$ is also purely imaginary, the $i$ factor therefore cancels off, and we will arrive at the displacement expression used in Eq. 24. 